
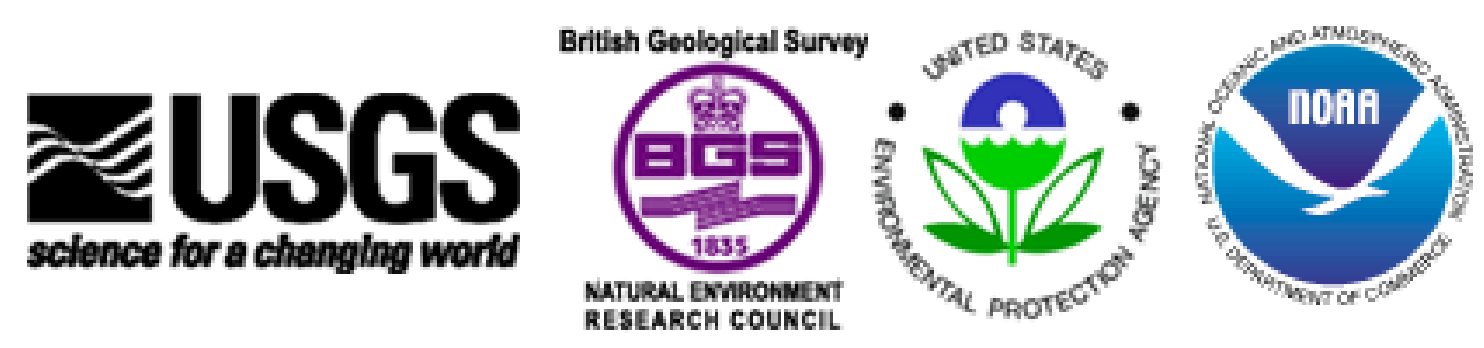

\title{
Measurement of Seafloor Radioactivity at the Farallon Islands Radioactive Waste Dump Site, California
}

By D.G. Jones', P.D. Roberts', J. Limburg', H. Karl', J.L. Chin', W.C. Shanks ${ }^{4}$, R. Hall, and D. Howard

\section{Open-File Report 01-62}

\section{1}

Any use of trade, firm, or product names is for descriptive purposes only and does not imply endorsement by the U.S. Government.

\section{U.S. Department of the Interior U.S. Geological Survey}

'British Geological Survey, Keyworth, Nottingham, NG12 5GG, UK

${ }^{2}$ Kernfysisch Versneller Instituut, Zernikelaan 25, 9747AA Groningen, The Netherlands

${ }^{3}$ U.S. Geological Survey, Menlo Park, CA 94025

${ }^{4}$ U.S. Geological Survey, Denver, CO 80225

${ }^{5}$ U.S. Environmental Protection Agency, San Francisco, CA 94105

${ }^{6}$ Gulf of the Farallones National Marine Sanctuary, San Francisco, CA 94129 


\section{Introduction}

Between 1946 and 1970, approximately 47,800 55-gallon drums, concrete blocks, and other containers of low-level radioactive waste were reportedly dumped offshore of San Francisco Bay. Three sites on the continental shelf and slope adjacent to the Farallon Islands were designated for the disposal of the waste (Joseph et al., 1971; NOAA, 1990; Noshkin et al., 1978; Waldichuk, 1960). According to the records, approximately 150 drums were deposited in a water depth of about $90 \mathrm{~m}, 3,600$ in a water depth of about $900 \mathrm{~m}$, and 44,000 in a nominal water depth of $1,800 \mathrm{~m}$. These sites are referred to here as the $90-\mathrm{m}$, $900-\mathrm{m}$, and 1,800-m sites, although the actual water depths at and around each site vary from the nominal values.

In reality, many of the drums were probably not disposed of at the specific sites. It is more likely that they litter a $1,400-\mathrm{km}^{2}$ area of sea floor, the Farallon Island Radioactive Waste Dump (Noshkin et al., 1978), defined by the irregular polygon on figure. 1.

Excluding tritium, an estimated total of some $540 \mathrm{TBq}(14,500 \mathrm{Ci})$ of thorium, uranium, transuranic, and other activation products, and mixed fission products were disposed of at the three locations (Joseph et al., 1971; Waldichuk, 1960). The precise amounts of individual radionuclides are not known. In addition to radioactive wastes, other wastes, including phenols, cyanides, mercury, beryllium and other heavy metals, dredge spoils, explosives, and garbage were also dumped in and around the area (NOAA, 1990).

Much of the Farallon Island Radioactive Waste Dump lies within the boundaries of the Gulf of the Farallones National Marine Sanctuary, which was designated in 1981 (fig. 1). In 1990, the U.S. Geological Survey (USGS) and the Gulf of the Farallones National Marine Sanctuary jointly surveyed part of the Farallon Island Radioactive Waste Dump with a sidescan-sonar system. The result of this survey (Karl et al., 1994), following subsequent computer enhancement of the data to assist in the identification of barrels, was the mapping of barrel locations over an area of $125 \mathrm{~km}^{2}$. The identification of the barrels was verified during the initial survey using an underwater camera/video and subsequently using the manned Navy submersible Sea Cliff and the unmanned Advanced Tethered Vehicle. Visual observations showed the barrels to be in all states of preservation, ranging from completely intact to completely disintegrated.

The Gulf of the Farallones and adjacent areas support a major commercial fishery. The area is also used extensively for sport fishing (NOAA, 1990). Fears of radioactive contamination have previously had an adverse impact on the fishery (E. Ueber, personal communication).

Discussions between the USGS and the British Geological Survey (BGS) led to a proposal to carry out a radioactivity survey of parts of the Farallon Islands Radioactive Waste Dump Site (fig. 1). This was done using the proven BGS towed seabed spectrometer (EEL) system (e.g., Jones, 1994; Miller et al., 1977), modified to operate in deeper water (Deep Tow EEL). The main aim was to obtain regional-scale information on sea floor radioactivity levels for parts of the site, particularly with reference to USGS mapping of barrel locations using sidescan sonar data (Karl et al., 1994). Previous radioactivity measurements had been restricted to the analyses of samples of water, sediments and biota (Dyer, 1976; Noshkin et al., 1978; PneumoDynamics Corporation, 1961; Schell and Sugai, 1980; Suchanek et al., 1996). In the case of the sediments, only a relatively small number of sites had been sampled, although some samples were collected from areas adjacent to barrels using submersibles (Dyer, 1976; Schell and Sugai, 1980).

The survey was carried out in April-May 1998 on the NOAA ship McArthur and involved interagency collaboration between the BGS, USGS, U.S. Environmental Protection Agency, National Oceanographic and Atmospheric Administration, and Gulf of the Farallones National Marine Sanctuary. Work was concentrated on the shallower parts of the site where commercial fishing occurs and where locations of barrels had been mapped. 


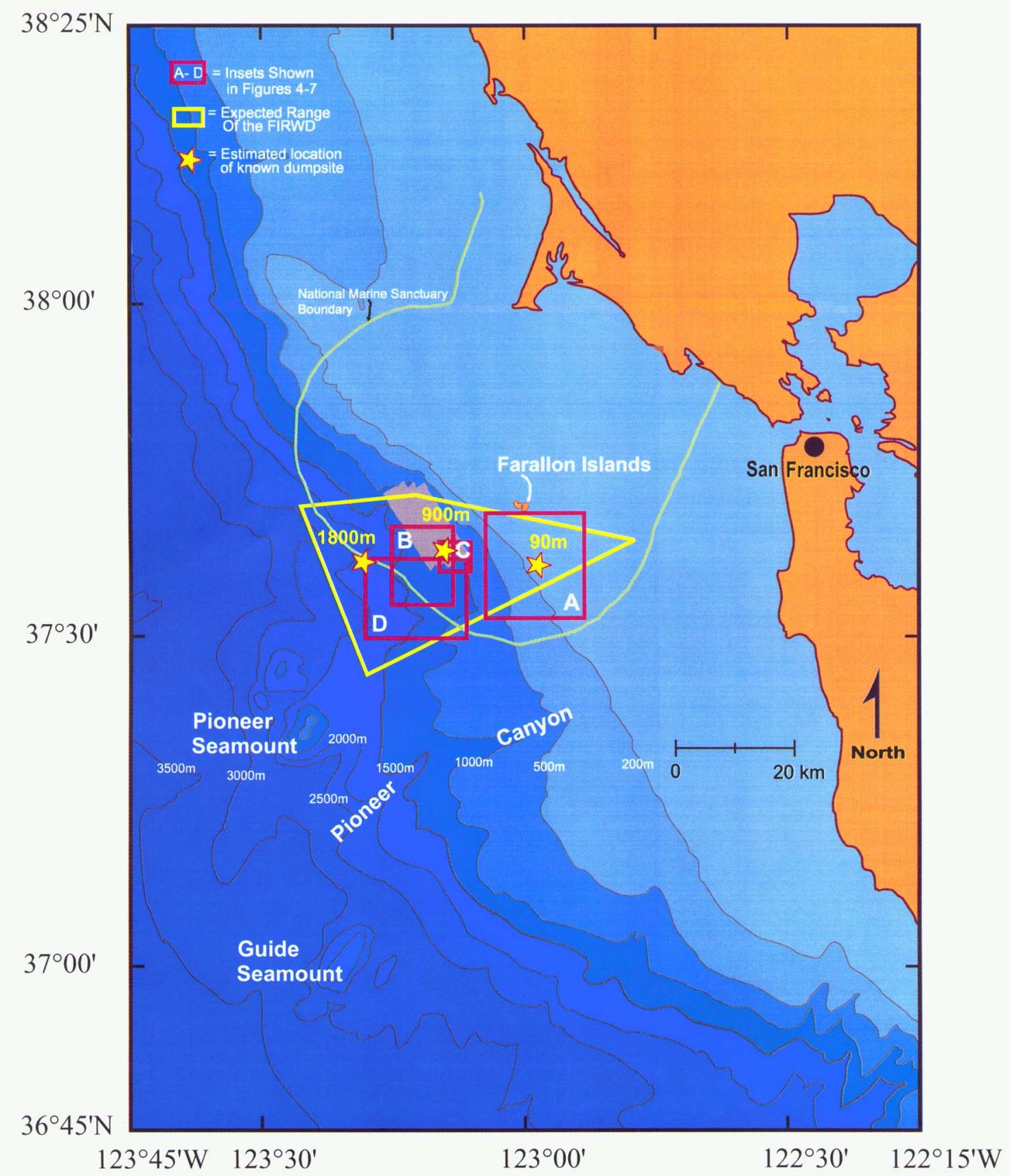

Figure 1. Farallon Islands Radioactive Waste Dump Site: Location map showing 90-, 900- and 1800m sites. Insets A-D show the areas covered by Figures 4-7, respectively.

\section{Seabed gamma-ray spectrometry}

The BGS EEL system (Jones, 1994; Miller et al., 1977) was developed for continental shelf use and has mostly been operated at depths less than $200 \mathrm{~m}$, although it was used briefly at $600 \mathrm{~m}$. Significant modifications were made to the system for the Farallon survey to cope with the much greater depths involved. This entailed uprating the pressure vessel that houses the detector to a 3,000-m capability, switching to digital data transmission to allow signal transmission through $6,000 \mathrm{~m}$ of cable, and completely updating the control electronics and data logging software. In addition, extra sensors were added to the 
seabed probe (c.f. de Meijer et al., 1997) to give pressure (=depth), temperature, and noise (=roughness) information (fig. 2). A 'saddleback' monitor was used to measure the strain on the towing cable to ensure that this stayed within safe working limits. This also incorporated a cable counter to keep track of the length of cable deployed.

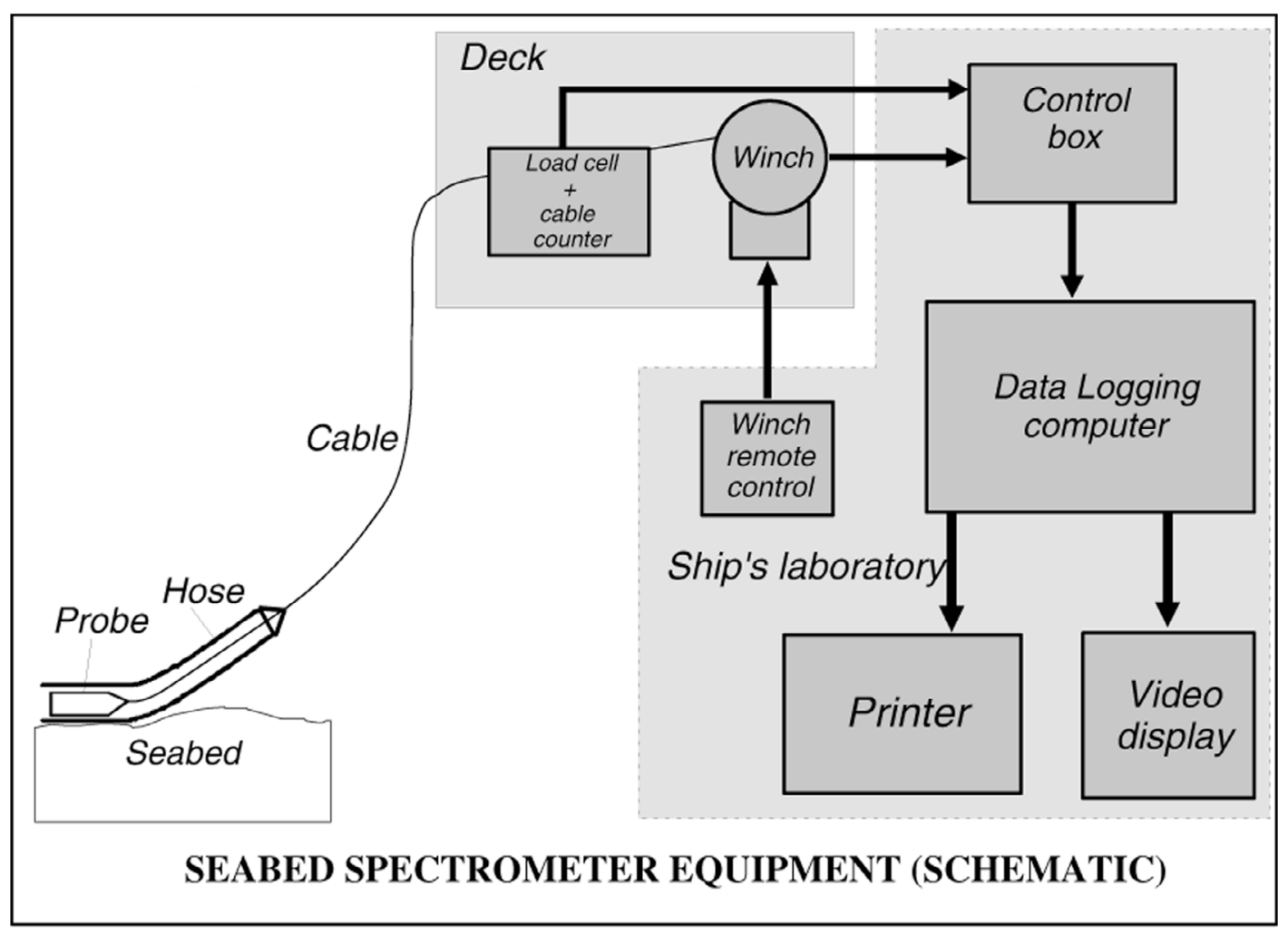

Figure 2. Schematic diagram of Deep Tow EEL system.

The detecting probe of the EEL is towed on the sea floor, protected inside a 30-m length of PVC hose (fig. 3). The gamma-ray detector used for the survey was a bismuth germanate (BGO) scintillation detector. When gamma rays interact with the detector, flashes of light (scintillations) are emitted. These are converted to voltage pulses and amplified and then sorted by pulse height. The intensity of the scintillations and the pulse height are proportional to the energy of the gamma rays. Hence the detector can measure the full gamma-ray spectrum, enabling contributions from natural and artificial radioactivity to be identified and quantified. The equipment measures the gamma emitters in the surficial sea floor material to an effective maximum depth of about $30 \mathrm{~cm}$.

The length of towing cable is altered, using a remote control, to maintain the probe on the seabed as the water depth changes or to accommodate changes in the speed of the survey vessel. A clear reduction in signal is seen if the probe loses contact with the sea bed. This is apparent in both the gamma-ray data and the sound signal and enables the detector, as far as possible, to be kept on the sea floor.

Although originally developed for geological mapping and mineral exploration (e.g., de Meijer et al., 1997; Jones et al., 1988a; Ringis et al., 1993), the equipment has subsequently been used for a variety of environmental surveys. These include the mapping of artificial radionuclides discharged from the Sellafield nuclear plant in the Irish Sea (Jones et al., 1988b; Miller et al., 1982). 


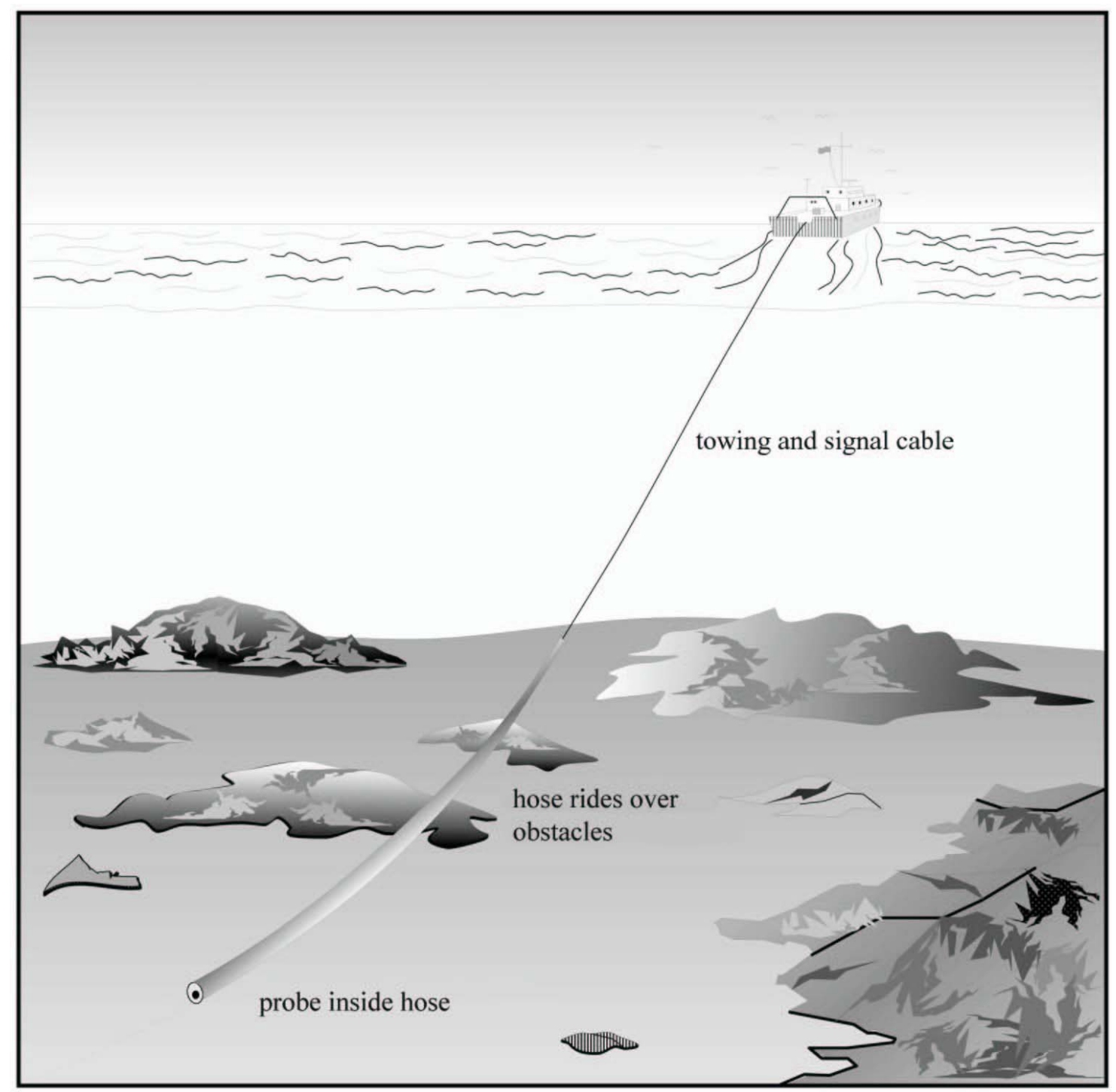

Figure 3. EEL method diagram.

\section{Survey operations}

The EEL was successfully deployed on the survey to a depth of $1,500 \mathrm{~m}$. Sets of lines were towed at both the 90-m and 900-m sites. Additional shorter tows were made through known barrel clusters at the 900-m site and lines extended from the 900-m site into deeper water adjacent to the 1,800-m site (see figure 1 for index map to areas covered). Bottom sediment samples were also collected. These related both to the EEL towing and to clusters of barrels, identified from the USGS sidescan data, in areas deemed to be too rugged for bottom towing.

The survey was planned so that work proceeded from shallow to deeper water; i.e., towing conditions for the EEL became progressively more hazardous. The aim was to maximise the likelihood of successful data collection.

Approximately 90 line-km of EEL data were towed on seven parallel traverses at the 90-m site, and nine sediment samples were collected with a Van Veen grab (fig. 4). The lines were oriented NW-SE, perpendicular to the direction of the swell, primarily because the ship could not maintain other headings in 
the prevailing sea conditions. Towing in the shallow water and predominately smooth seafloor conditions was relatively straightforward.

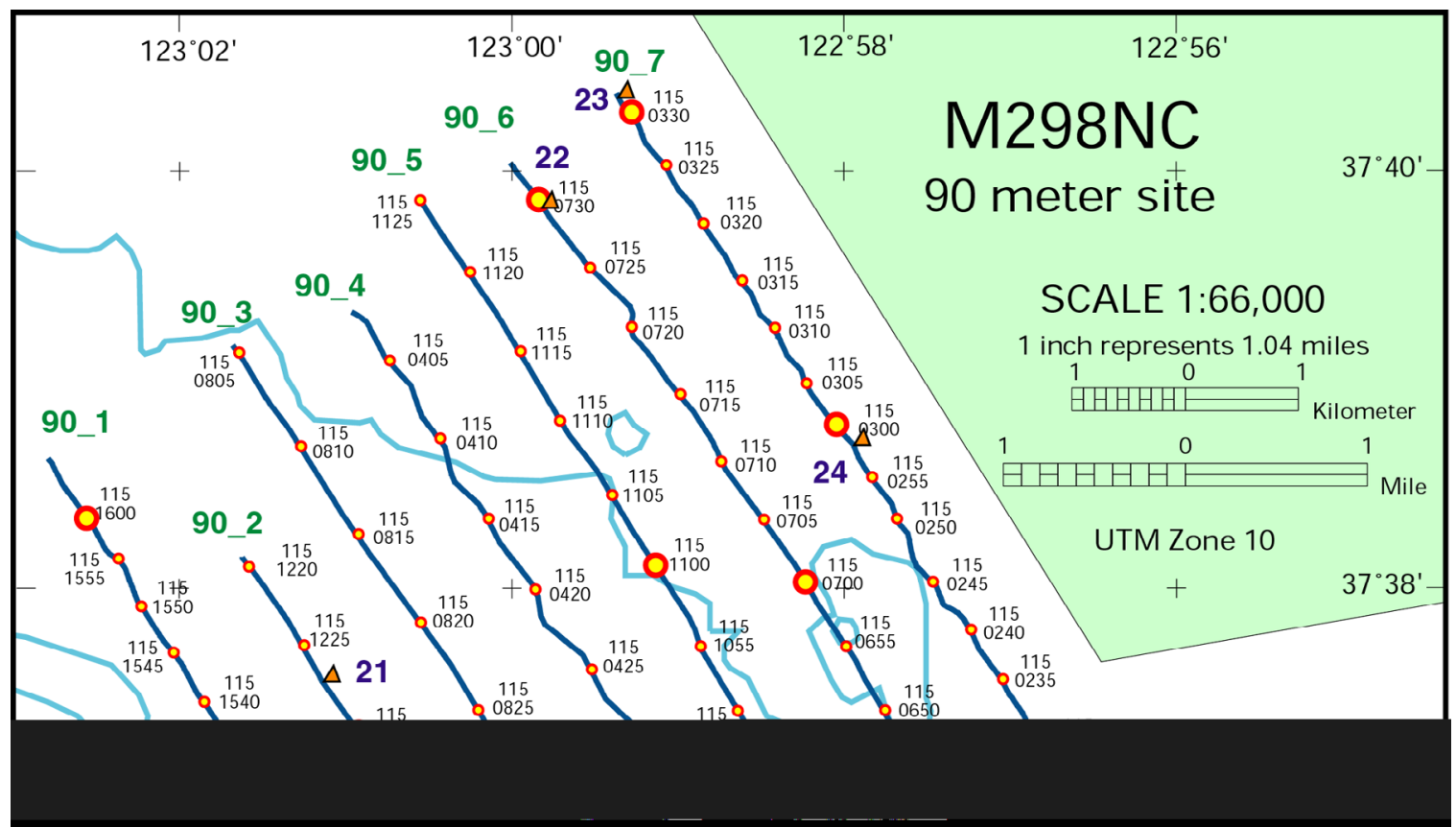

Figure 4. Survey lines and sample locations at 90-m site (e.g., $\Delta 20)$. Contours show depths (m) below mean sea level. 
A further set of seven NW-SE lines, totalling some 60 line-km, was surveyed at the $900-\mathrm{m}$ site (fig.

5). These were sited mainly on a broad spur between two submarine canyons, where seafloor

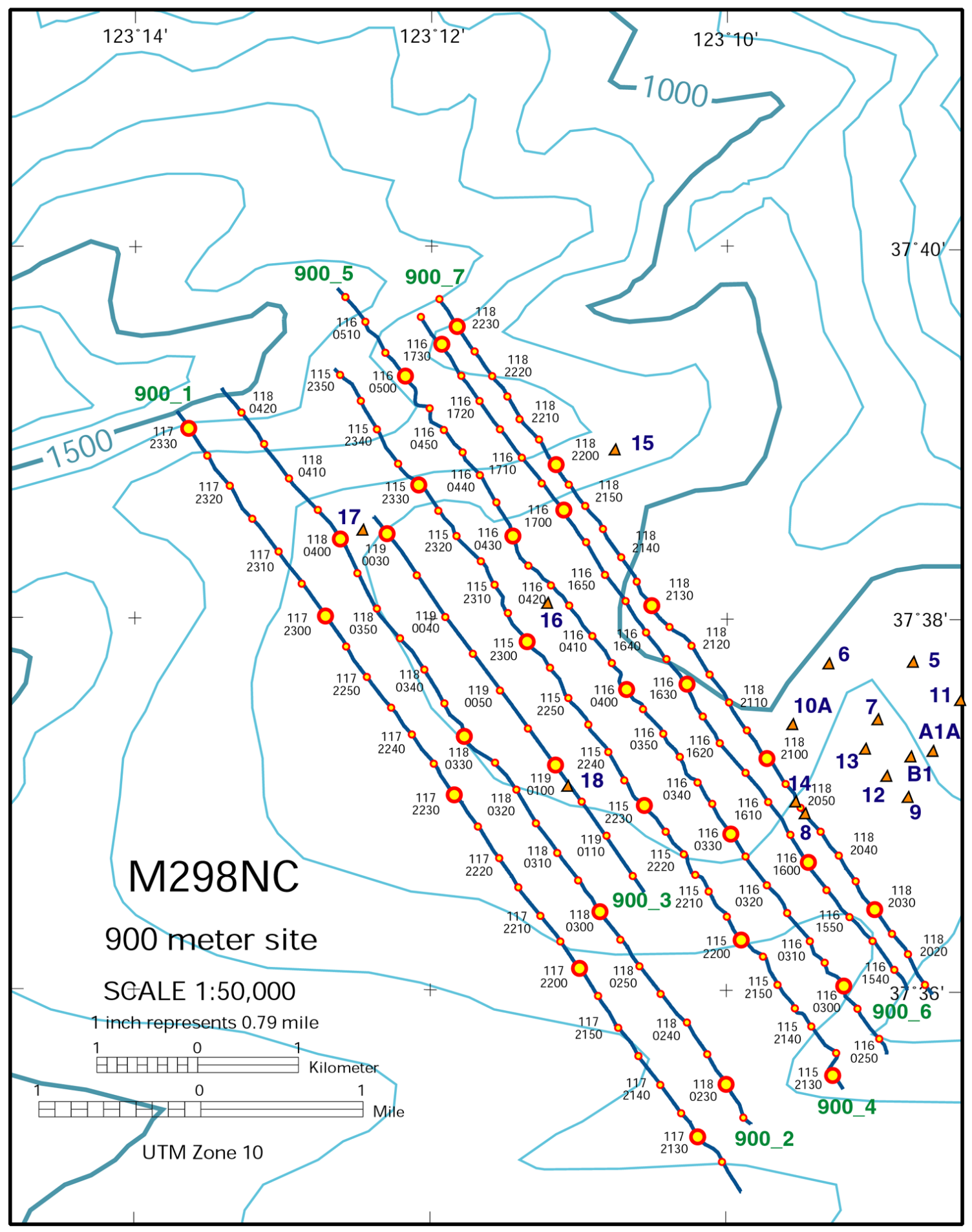

Figure 5. Survey lines and sample locations at 900-m site (e.g., $\Delta 15)$. Contours show depths (m) below mean sea level 
gradients were not too extreme. Two short traverses (about. 5.5 line-km) were made through known barrel clusters (fig. 6). Six sediment samples were collected with the grab related to lines towed at the $900-\mathrm{m}$ site as well as a further 10 samples in the vicinity of barrel clusters that were not towed with the EEL (fig. 5).

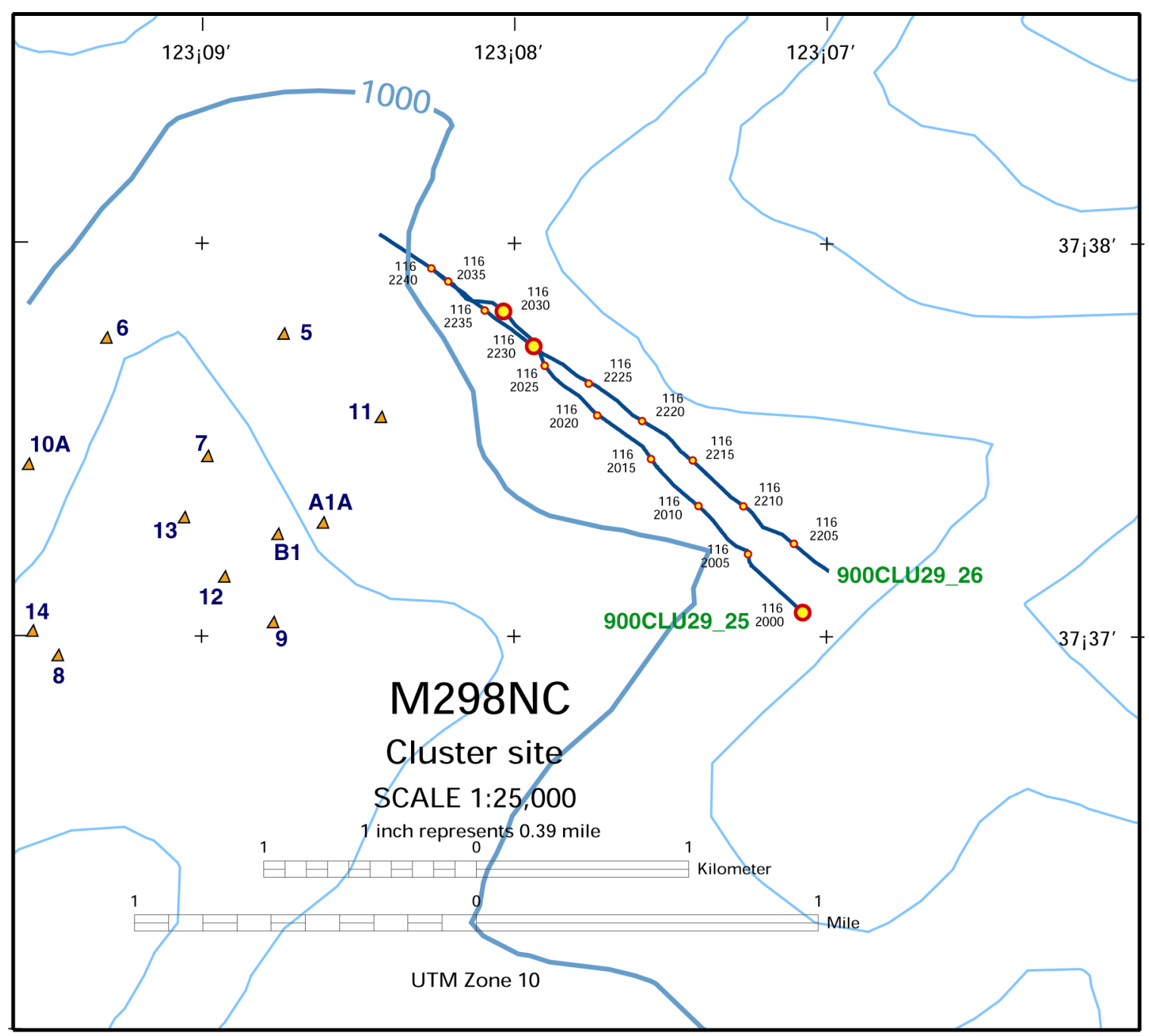

Figure 6. Survey lines and sample locations (e.g. $\Delta 12)$ at the 'Cluster' site. Contours show depths (m) below mean sea level.

Two lines (about 18 line-km) were run from the 900-m site into deeper water adjacent to the 1,800-m site (fig. 7). These were run roughly perpendicular to the main 900-m traverses, in improved sea conditions, and reached a maximum depth of $1,500 \mathrm{~m}$.

Grab sampling was restricted, by the length of cable available on the ship's sampling winch, to a maximum water depth of about 1,200 $\mathrm{m}$. Thus no samples were collected from the deeper parts of the area surveyed with the EEL.

The EEL radiometric data were collected continuously over 5-s intervals with the full gamma-ray spectrum being displayed on the logging screen over the previous 10 minutes and integrated over the entire 


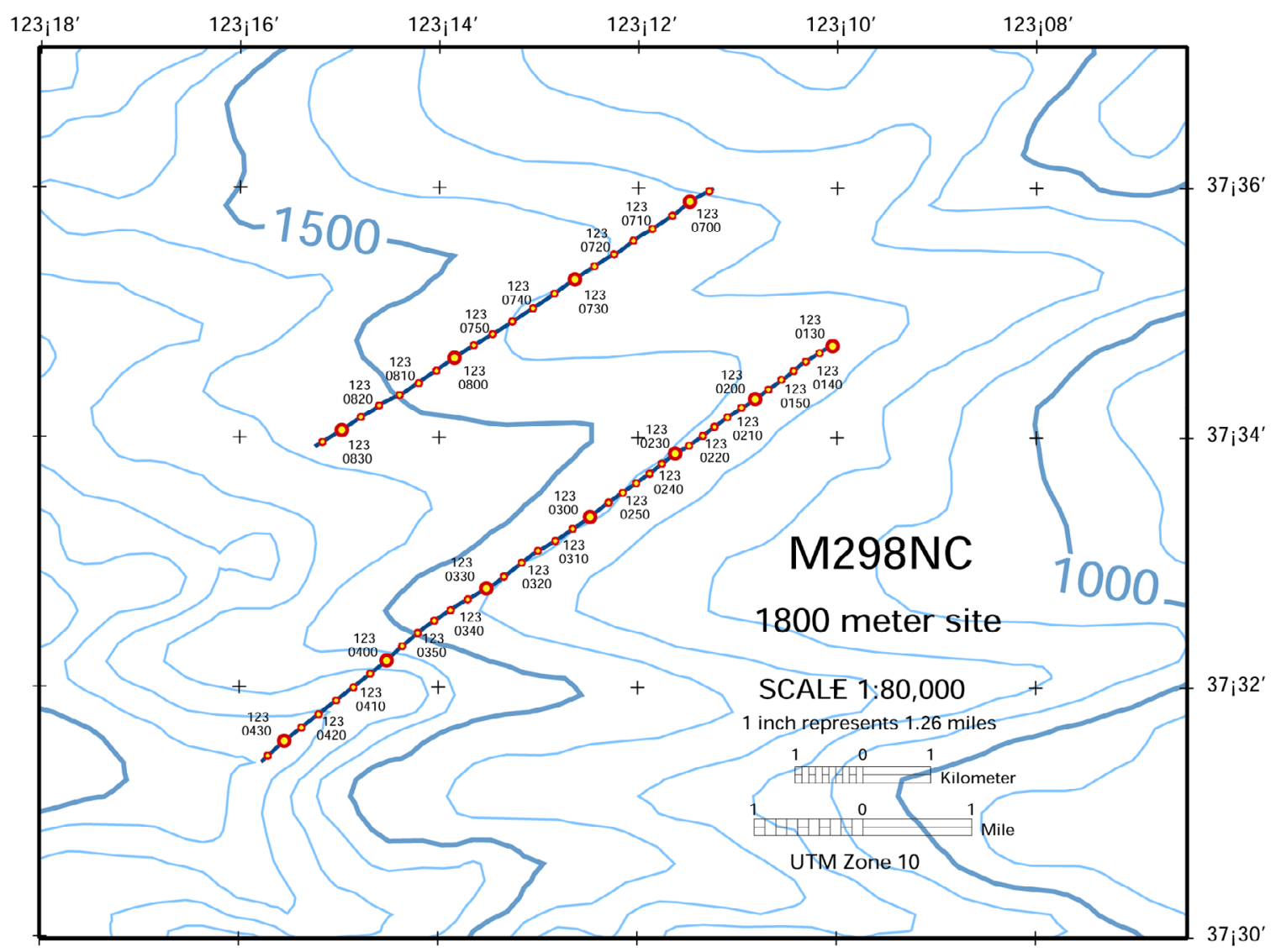

Figure 7. 1 Survey lines at 1,800-m site. Contours show depths (m) below mean sea level.line. At the typical towing speed of approximately 2 knots $\left(3.6 \mathrm{kmh}^{-1}\right)$, a 5 -s interval represents the coverage of about $5 \mathrm{~m}$ of seabed.

line. At the typical towing speed of approximately 2 knots $\left(3.6 \mathrm{kmh}^{-1}\right)$, a 5 -s interval represents the coverage of about $5 \mathrm{~m}$ of seabed.

\section{Laboratory analyses}

The gamma-emitting radionuclides in the sediment samples were analysed (in BGS and USGS laboratories) using high-resolution gamma spectrometry in low background counting facilities. The two sets of data are generally in good agreement. The BGS results are summarised in table 1 . The Am and $\mathrm{Pu}$ content of a selection of samples was also investigated at BGS by alpha spectrometry, following extraction and concentration, as outlined in Jones et al. (1999). The results are summarised in table 2.

Grain size analysis of the sediments (summarised in table 3) was carried out at the USGS facility in Menlo Park. Coarse fractions were separated by wet sieving, intermediate fractions by using Rapid Settling Analysis in settling tubes, and fine fractions by using a Micromeritics SediGraph 5100 particle-size analyser.

The general geochemistry of the samples was undertaken at USGS Denver by ICP-MS following an acid digestion (see Briggs and Meier, 1999 for details). This had the dual purpose of providing background geochemical information on the sediments and their variability and of assessing whether any nonradioactive contaminants were present. The results are given in table 4.

Quality Assurance/Quality Control of the laboratory determinations was ensured by the use of international standards or other accredited reference materials and by analyzing duplicate samples and blanks. The laboratories of the BGS and USGS also took part in interlaboratory comparison exercises. 
Table 1. Radionuclide data for sediment samples (in $\mathrm{Bqkg}^{-1}$ ) by gamma-ray spectrometry at BGS (errors are $1 \sigma$ counting errors)

\begin{tabular}{|c|c|c|c|c|c|c|c|c|c|c|c|}
\hline Site & \begin{tabular}{|l} 
Sample \\
No.
\end{tabular} & ${ }^{241} \mathrm{Am}$ & $\begin{array}{l}241 \mathrm{Am} \\
\text { Error }\end{array}$ & ${ }^{137} \mathrm{Cs}$ & $\begin{array}{l}{ }^{137} \text { Cs } \\
\text { Error }\end{array}$ & ${ }^{40} K$ & $\begin{array}{l}{ }^{40} \mathrm{~K} \\
\text { Error }\end{array}$ & $\mathrm{U}\left({ }^{214} \mathbf{B i}\right)$ & $\begin{array}{l}\mathbf{U} \\
\text { Error }\end{array}$ & $\operatorname{Th}\left({ }^{208} \mathrm{Tl}\right)$ & \begin{tabular}{|l|} 
Th \\
Error
\end{tabular} \\
\hline \multirow[t]{12}{*}{ Cluster' } & A1A & $<0.5$ & & 0.8 & 0.4 & 581.6 & 11.2 & 44.5 & $\begin{array}{ll}1.3 \\
\end{array}$ & 27.6 & 1.5 \\
\hline & B1 & 3.5 & 0.6 & 1.9 & 0.8 & 582.1 & 16.4 & 49.5 & 2.0 & 24.9 & 2.1 \\
\hline & $10 \mathrm{~A}$ & 1.2 & 0.3 & 0.9 & 0.3 & 578.8 & 13.9 & 24.1 & 1.4 & 20.2 & 1.7 \\
\hline & 5 & $<0.5$ & & 4.2 & 0.8 & 578.8 & 16.5 & 42.5 & 1.9 & 27.9 & 2.3 \\
\hline & 6 & $<0.5$ & & 1.8 & 0.8 & 560.2 & 15.5 & 43.7 & 1.9 & 24.8 & 2.2 \\
\hline & 7 & $<0.5$ & & 2.6 & 0.7 & 567.5 & 15.3 & 44.6 & 1.7 & 24.4 & 2.0 \\
\hline & 8 & $<0.5$ & & $<0.5$ & & 591.0 & 19.8 & 42.7 & 2.5 & 21.6 & 3.2 \\
\hline & 9 & $<0.5$ & & 3.8 & 0.8 & 571.1 & 17.5 & 43.4 & 2.0 & 21.3 & 2.3 \\
\hline & 11 & 2.5 & 0.4 & 1.7 & 0.4 & 612.3 & 12.7 & 36.2 & \begin{tabular}{|l|}
1.4 \\
\end{tabular} & 26.5 & 1.5 \\
\hline & 12 & $<0.5$ & & 2.6 & 0.7 & 578.8 & 14.7 & 43.4 & 1.6 & 21.6 & 2.0 \\
\hline & 13 & $<0.5$ & & 2.6 & 0.7 & 579.2 & 14.9 & 42.3 & 1.8 & 23.4 & 1.9 \\
\hline & 14 & 2.6 & 0.4 & 3.5 & 0.4 & 574.6 & 10.4 & 46.4 & 1.3 & 21.9 & 1.3 \\
\hline \multirow[t]{4}{*}{$900 \mathrm{~m}$} & 15 & $<1.0$ & & $<0.5$ & & 616.4 & 12.9 & 17.7 & 1.2 & 11.8 & 1.6 \\
\hline & 16 & $<1.0$ & & $<0.5$ & & 574.7 & 12.4 & 21.2 & 1.2 & 15.4 & 1.5 \\
\hline & 17 & $<1.0$ & & $<0.5$ & & 584.4 & 13.4 & 24.2 & 1.3 & 22.0 & 1.6 \\
\hline & 18 & $<0.5$ & & 1.5 & 0.3 & 543.6 & 13.4 & 36.4 & 1.6 & 23.2 & 2.0 \\
\hline \multirow[t]{9}{*}{$90 \mathrm{~m}$} & 19 & $<0.5$ & & 1.3 & 0.3 & 522.0 & 7.9 & 29.6 & 1.1 & 29.8 & 1.0 \\
\hline & 20 & $<0.5$ & & 1.0 & 0.5 & 451.0 & 10.9 & 18.7 & 1.0 & 16.4 & 1.2 \\
\hline & 21 & $<0.5$ & & 1.2 & 0.4 & 482.0 & 8.5 & 18.2 & 0.8 & 18.1 & 1.1 \\
\hline & 22 & $<0.5$ & & $<0.5$ & & 420.0 & 10.3 & 8.2 & 1.0 & 21.2 & 1.3 \\
\hline & 23 & $<0.5$ & & $<0.5$ & & $\begin{array}{l}193.0 \\
\end{array}$ & 6.4 & 3.8 & 0.8 & \begin{tabular}{|c|}
6.1 \\
\end{tabular} & 0.9 \\
\hline & 24 & $<0.5$ & & 2.1 & 0.4 & 466.0 & 10.9 & 16.4 & 1.1 & 15.6 & 1.2 \\
\hline & 25 & $<0.5$ & & 1.9 & 0.4 & 480.0 & 11.9 & 18.4 & 1.0 & 17.1 & 1.3 \\
\hline & 26 & $<0.5$ & & 1.3 & 0.3 & 506.0 & 7.6 & 21.3 & 0.7 & 21.9 & 0.8 \\
\hline & 27 & $<0.5$ & & 1.0 & \begin{tabular}{|l|}
0.3 \\
\end{tabular} & 476.0 & 10.8 & 18.7 & 1.0 & \begin{tabular}{|l|}
17.3 \\
\end{tabular} & 1.3 \\
\hline
\end{tabular}


Table 2. Radionuclide data for sediment samples (in $\mathrm{Bqkg}^{-1}$ ) by alpha spectrometry at BGS (errors are $1 \sigma$ counting errors)

\begin{tabular}{|c|c|c|c|c|c|c|c|c|c|c|}
\hline Site & Field No. & Longitude & Latitude & $\begin{array}{l}\text { Depth } \\
\text { (m) }\end{array}$ & ${ }^{239 / 240} \mathrm{Pu}$ & $\begin{array}{l}{ }^{239 / 240} \mathrm{Pu} \\
\text { error }\end{array}$ & ${ }^{238} \mathrm{Pu}$ & 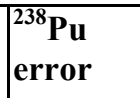 & ${ }^{241} \mathrm{Am}$ & $\stackrel{\text { error }}{241} \mathrm{Am}$ \\
\hline \multirow[t]{12}{*}{ Cluster' } & A1A & -123.14352 & 37.62143 & 1450 & & & & & & \\
\hline & B1 & -123.14588 & 37.62096 & 1150 & 2.6 & 0.1 & $<0.1$ & & 1.8 & 0.2 \\
\hline & $10 \mathrm{~A}$ & -123.15921 & 37.62384 & 1100 & & & & & & \\
\hline & 5 & -123.14562 & 37.62943 & 1100 & 3.1 & 0.2 & 0.3 & 0.07 & 2 & 0.07 \\
\hline & 6 & -123.15510 & 37.62925 & 1100 & & & & & & \\
\hline & 7 & -123.14967 & 37.62427 & 1150 & 2.8 & 0.2 & $<0.1$ & & 1.2 & 0.9 \\
\hline & 8 & -123.15765 & 37.61577 & 1180 & & & & & & \\
\hline & 9 & -123.14616 & 37.61723 & 1150 & & & & & & \\
\hline & 11 & -123.14038 & 37.62593 & 1060 & & & & & & \\
\hline & 12 & $\mid-123.14868$ & 37.61915 & 1150 & & & & & & \\
\hline & 13 & $\mid-123.15097$ & 37.62163 & 1150 & & & & & & \\
\hline & 14 & -123.15897 & 37.61677 & 1120 & 2.1 & 0.1 & $<0.1$ & & $<0.1$ & \\
\hline \multirow[t]{4}{*}{$900 \mathrm{~m}$} & 15 & -123.17923 & 37.64832 & 1050 & & & & & & \\
\hline & 16 & -123.18697 & 37.63455 & 1015 & & & & & & \\
\hline & 17 & \begin{tabular}{|l|l|}
-123.20768 \\
\end{tabular} & 37.64116 & 1080 & 0.8 & 0.04 & $<0.1$ & & $<0.1$ & \\
\hline & 18 & -123.18455 & 37.61825 & 1100 & 1.1 & 0.06 & $<0.1$ & & $<0.1$ & \\
\hline \multirow[t]{9}{*}{$90 \mathrm{~m}$} & 19 & -123.00136 & 37.60260 & 136 & 0.6 & 0.04 & $<0.1$ & & $<0.1$ & \\
\hline & 20 & -123.00207 & 37.62037 & 118 & & & & & & \\
\hline & 21 & -123.01758 & 37.62567 & 123 & & & & & & \\
\hline & 22 & -122.99587 & 37.66318 & 73 & & & & & & \\
\hline & 23 & -122.98837 & 37.67187 & 58 & & & & & & \\
\hline & 24 & -122.96485 & 37.64437 & 91 & 0.8 & 0.05 & $<0.1$ & & 0.2 & 0.04 \\
\hline & 25 & \begin{tabular}{|l|l}
-122.96890 \\
\end{tabular} & 37.59508 & 114 & & & & & & \\
\hline & 26 & $\mid-122.98218$ & 37.56576 & 131 & & & & & & \\
\hline & 27 & -122.90722 & 37.57253 & 100 & & & & & & \\
\hline
\end{tabular}


Table 3. Summary of grain size data for sediment samples (data produced by sieving, settling tubes, and SediGraph at USGS)

\begin{tabular}{|c|r|r|r|r|r|}
\hline Sample & Gravel (\%) & Sand (\%) & Mud (\%) & \multicolumn{1}{|c|}{ Silt (\%) } & \multicolumn{1}{c|}{ Clay (\%) } \\
\hline A1A & 0.0 & 6.5 & 93.5 & 55.7 & 37.8 \\
\hline B1 & 0.0 & 10.5 & 89.5 & 50.8 & 38.8 \\
\hline $\mathbf{1 0 A}$ & 0.0 & 59.7 & 40.3 & 22.1 & 18.2 \\
\hline $\mathbf{5}$ & 0.0 & 55.8 & 44.2 & 29.5 & 14.7 \\
\hline $\mathbf{6}$ & 0.0 & 17.6 & 82.4 & 44.3 & 38.1 \\
\hline $\mathbf{7}$ & 0.0 & 44.4 & 55.6 & 35.4 & 20.2 \\
\hline $\mathbf{8}$ & nd & nd & nd & nd & nd \\
\hline $\mathbf{9}$ & 0.0 & 6.3 & 9.8 & 55.2 & 38.6 \\
\hline $\mathbf{1 1}$ & 0.0 & 7.0 & 93.0 & 53.8 & 39.2 \\
\hline $\mathbf{1 2}$ & 0.0 & 29.1 & 70.9 & 41.9 & 29.0 \\
\hline $\mathbf{1 3}$ & 0.0 & 17.8 & 82.3 & 45.2 & 37.1 \\
\hline $\mathbf{1 4}$ & 0.0 & 6.0 & 94.0 & 54.1 & 39.9 \\
\hline $\mathbf{1 5}$ & 0.0 & 67.0 & 33.0 & 18.6 & 14.3 \\
\hline $\mathbf{1 6}$ & 0.0 & 72.0 & 28.0 & 16.8 & 11.2 \\
\hline $\mathbf{1 7}$ & 0.0 & 33.5 & 66.6 & 36.3 & 30.2 \\
\hline $\mathbf{1 8}$ & 0.0 & 20.9 & 79.1 & 46.6 & 32.6 \\
\hline $\mathbf{1 9}$ & 0.0 & 93.1 & 6.9 & 4.4 & 2.6 \\
\hline $\mathbf{2 0}$ & 0.0 & 77.0 & 23.0 & 17.7 & 5.3 \\
\hline $\mathbf{2 1}$ & 0.0 & 68.4 & 31.6 & 27.7 & 3.9 \\
\hline $\mathbf{2 2}$ & 0.0 & 98.0 & 2.0 & 2.0 & 0.0 \\
\hline $\mathbf{2 3}$ & 0.0 & 98.6 & 1.4 & 1.4 & 0.0 \\
\hline $\mathbf{2 4}$ & 0.0 & 60.4 & 39.6 & 29.2 & 10.4 \\
\hline $\mathbf{2 5}$ & 0.0 & 66.0 & 34.0 & 27.1 & 6.9 \\
\hline $\mathbf{2 6}$ & 0.0 & 87.7 & 12.4 & 6.9 & 5.4 \\
\hline $\mathbf{2 7}$ & 0.0 & 80.6 & 19.4 & 15.1 & 4.4 \\
\hline & & & & & \\
\hline
\end{tabular}


Table 4. Summary of geochemical data for sediment samples (analyses by ICP-MS after acid digestion at USGS Denver)

\begin{tabular}{|c|c|c|c|c|c|c|c|c|c|c|c|c|c|c|c|c|c|c|c|c|c|c|c|c|}
\hline Sample & Al & $\mathbf{C a}$ & $\mathrm{Fe}$ & $\mathbf{K}$ & $\mathbf{M g}$ & $\mathbf{N a}$ & $\mathbf{A g}$ & As & Ba & $\mathrm{Be}$ & Bi & $\mathbf{C d}$ & $\mathrm{Ce}$ & Co & $\mathbf{C r}$ & $\mathbf{C u}$ & Dy & $\mathbf{E r}$ & $\mathbf{E u}$ & Ga & Gd & Ge & Ho & $\mathbf{L a}$ \\
\hline & $\%$ & $\%$ & $\%$ & $\%$ & $\%$ & $\%$ & ppm & ppm & ppm & ppm & ppm & ppm & ppm & ppm & ppm & ppm & ppm & ppm & ppm & ppm & ppm & ppm & ppm & ppm \\
\hline$\widehat{\mathrm{A} 1 \mathrm{~A}}$ & 7.2 & 1.9 & 3.5 & 1.6 & 1.8 & 2 & 0.26 & 7.4 & 590 & 1.5 & 0.2 & 1 & 46 & 10 & 150 & 30 & 4.4 & 2.7 & $\mid 0.96$ & 17 & 4.2 & 1.1 & 0.88 & 26 \\
\hline B1 & 7.2 & 1.8 & 3.7 & \begin{tabular}{|l|}
1.6 \\
\end{tabular} & 1.7 & \begin{tabular}{|l|}
1.7 \\
\end{tabular} & 0.36 & 7.5 & 680 & \begin{tabular}{|l|}
1.4 \\
\end{tabular} & 0.1 & 1 & 55 & 11 & 150 & 30 & 3.9 & 2.6 & \begin{tabular}{|l|}
0.99 \\
\end{tabular} & 17 & 4.2 & 1.3 & \begin{tabular}{|l|}
0.89 \\
\end{tabular} & 29 \\
\hline $10 \mathrm{~A}$ & 6.4 & 1.3 & 6.2 & 1.8 & 1.6 & 1.9 & 0.27 & 6.9 & 560 & 1.6 & $<0.05$ & 0.7 & 35 & \begin{tabular}{|l|} 
\\
\end{tabular} & 140 & 20 & 2.9 & 1.9 & \begin{tabular}{|l|}
0.8 \\
\end{tabular} & 14 & 3 & 1.9 & 0.62 & \begin{tabular}{|l|}
17 \\
\end{tabular} \\
\hline 5 & 7.6 & 1.7 & 3.7 & 1.7 & 2 & 2.2 & 0.36 & 7.5 & 610 & 1.5 & 0.1 & 1 & 49 & 11 & 150 & 30 & 3.9 & 2.6 & \begin{tabular}{|l|}
0.98 \\
\end{tabular} & 17 & 4.2 & 1.2 & $\mid 0.89$ & 24 \\
\hline 6 & 7.1 & 2.8 & 3.4 & 1.5 & 1.8 & 2.2 & 0.36 & 8 & 580 & 1.2 & 0.1 & 1 & 45 & 11 & 150 & 30 & 3.9 & 2.3 & 0.96 & 15 & 4 & 1.1 & 0.81 & 23 \\
\hline 7 & 7.8 & 1.8 & 3.6 & 1.6 & 2 & 2 & 0.35 & 7.4 & 560 & 1.4 & 0.1 & 1.1 & 42 & 11 & 150 & 30 & 3.7 & 2.4 & 0.93 & 17 & 3.8 & 1 & \begin{tabular}{|l|l}
0.8 \\
\end{tabular} & 22 \\
\hline 8 & 7.3 & 1.9 & 3.7 & 1.6 & 1.9 & 1.8 & 0.4 & 7.3 & \begin{tabular}{|l|}
670 \\
\end{tabular} & 1.4 & 0.1 & 1 & 47 & 11 & 150 & 30 & 4.3 & 2.7 & \begin{tabular}{|l|}
0.99 \\
\end{tabular} & 17 & 4 & 1.2 & 0.92 & 25 \\
\hline 9 & 7.3 & 1.9 & 3.6 & 1.6 & 1.8 & 2.2 & 0.4 & 7.9 & 570 & 1.2 & 0.1 & 1 & 45 & 11 & 150 & 31 & 4.2 & 2.7 & \begin{tabular}{|l|}
0.97 \\
\end{tabular} & 16 & 4 & 1.2 & \begin{tabular}{|l|}
0.84 \\
\end{tabular} & 23 \\
\hline$\overline{11}$ & 7.8 & 1.6 & 3.7 & 1.6 & 1.9 & 2 & 0.23 & 7 & 580 & 1.3 & 0.1 & 1 & 48 & 11 & 150 & 30 & 3.9 & 2.4 & 0.98 & 17 & 4 & 1.3 & 0.8 & 24 \\
\hline 12 & 7.3 & 2.1 & 3.6 & 1.8 & 1.7 & 2.3 & 0.31 & 8 & 610 & 1.3 & 0.09 & 1.1 & 46 & 11 & 150 & 30 & 3.9 & 2.5 & 1 & 17 & 4.3 & 1.2 & \begin{tabular}{|l|}
0.84 \\
\end{tabular} & 25 \\
\hline 13 & 7 & 1.9 & 3.6 & 1.8 & 1.8 & 2.3 & 0.29 & 8.3 & 620 & 1.2 & 0.08 & 1.2 & 45 & 11 & 140 & 30 & 3.6 & 2.5 & \begin{tabular}{|l|}
0.88 \\
\end{tabular} & 16 & 3.7 & 1.2 & 0.86 & 24 \\
\hline 14 & 7.4 & 2.1 & 3.8 & 1.6 & 2 & 2.1 & 0.35 & 7.8 & 680 & 1.3 & 0.1 & 0.8 & 46 & 11 & 160 & 31 & 4 & 2.5 & \begin{tabular}{|l|}
0.97 \\
\end{tabular} & 17 & 4.2 & 1.2 & \begin{tabular}{|l|}
0.91 \\
\end{tabular} & 28 \\
\hline 15 & 5.1 & 1.1 & 9.1 & 2 & 1.7 & 1.9 & 0.28 & 7 & 430 & 2.2 & $<0.05$ & 0.3 & 31 & 6.8 & 160 & 20 & 2.7 & 1.6 & 0.6 & 12 & 2.7 & 2.4 & 0.53 & 15 \\
\hline 16 & 7.2 & 1.6 & 4.2 & 1.8 & 1.5 & 2.1 & 0.18 & 5.6 & 580 & 1.2 & 0.06 & 0.5 & 42 & 9 & 150 & 20 & 3.7 & 2.2 & \begin{tabular}{|l|}
0.93 \\
\end{tabular} & 15 & 3.8 & 1.5 & \begin{tabular}{|l|}
0.81 \\
\end{tabular} & 21 \\
\hline 17 & 7.6 & 1.5 & 4.8 & 1.9 & 1.8 & 2.2 & 0.17 & 7.1 & 640 & 1.5 & 0.06 & 0.5 & 50 & 11 & 160 & 20 & 3.8 & 2.6 & 1 & 17 & 4 & 1.6 & 0.81 & 26 \\
\hline $\mid 18$ & 7.9 & 2 & 3.7 & 1.8 & 1.8 & 2 & 0.19 & 7 & 680 & 1.4 & 0.07 & 1.3 & 44 & 11 & 140 & 20 & 4 & 2.4 & 1 & 18 & 3.8 & 1.3 & 0.85 & 21 \\
\hline 19 & 7.2 & 2.8 & 3 & 1.7 & 1.4 & 2.2 & 0.14 & 6.7 & 600 & \begin{tabular}{|l|l|}
1.4 \\
\end{tabular} & $<0.05$ & 0.8 & 55 & 12 & 120 & 8 & 4 & 2.7 & 1.1 & 13 & 4.7 & 0.9 & 0.93 & 27 \\
\hline 20 & 7.4 & 1.9 & 2.7 & 1.6 & 1.4 & 2.4 & 0.08 & 6.1 & 640 & 1 & $<0.05$ & 3.6 & 44 & 8.3 & 130 & 10 & 3.4 & 2.2 & 1 & 14 & 3.5 & 1.1 & 0.76 & 20 \\
\hline 21 & 7.6 & 1.9 & 2.8 & \begin{tabular}{|l|}
1.6 \\
\end{tabular} & 1.4 & 2.3 & 0.05 & \begin{tabular}{|l|}
5.8 \\
\end{tabular} & 590 & 1.3 & $<0.05$ & 3 & 41 & 8.6 & 130 & 10 & $\begin{array}{l}3.7 \\
\end{array}$ & 2.4 & 0.97 & 13 & 3.9 & 1.1 & \begin{tabular}{|l|l|}
0.77 \\
\end{tabular} & 22 \\
\hline 22 & \begin{tabular}{|l|}
5.8 \\
\end{tabular} & 13 & 1.1 & $\mid 1.1$ & 0.43 & 2.5 & 0.02 & 5 & 710 & 1 & $<0.05$ & $\mid 0.1$ & 62 & 1.4 & 17 & 3 & 2 & $\mid 1.1$ & 0.73 & 10 & 2.5 & 0.5 & 0.42 & 32 \\
\hline 23 & 1.4 & 32 & 0.71 & 0.36 & \begin{tabular}{|l|}
0.48 \\
\end{tabular} & 0.98 & $<0.02$ & 6.1 & 240 & 0.3 & $<0.05$ & 0.2 & 15 & 0.5 & 9 & $<3$ & 0.98 & 0.58 & 0.25 & 2.7 & 1.1 & 0.1 & 0.21 & \begin{tabular}{|l|}
8.7 \\
\end{tabular} \\
\hline 24 & \begin{tabular}{|l|l}
6.8 \\
\end{tabular} & 1.5 & 2.6 & 1.5 & 1.2 & 2.2 & $<0.02$ & 6 & 670 & \begin{tabular}{|l|}
1.5 \\
\end{tabular} & $<0.05$ & 3.2 & 39 & 7.2 & 120 & 10 & 3 & 2 & 0.88 & 13 & 3.3 & 1 & \begin{tabular}{|l|l|}
0.69 \\
\end{tabular} & 19 \\
\hline 25 & 7.6 & 2 & 3.1 & \begin{tabular}{|l|}
1.6 \\
\end{tabular} & 1.5 & 2.4 & $<0.02$ & \begin{tabular}{|l|l|}
5.7 \\
\end{tabular} & 660 & 1.3 & $<0.05$ & 3.6 & 45 & 9.5 & 140 & 10 & 3.6 & 2.3 & 1 & 14 & 3.9 & 1 & \begin{tabular}{|l|l|}
0.77 \\
\end{tabular} & 22 \\
\hline 26 & 7.7 & 2.4 & 3.2 & 1.8 & \begin{tabular}{|l|}
1.6 \\
\end{tabular} & 2.1 & $<0.02$ & 6 & 600 & \begin{tabular}{|l|l|}
1.4 \\
\end{tabular} & $<0.05$ & 2.5 & 56 & 11 & 140 & 10 & 4 & 2.6 & 1.2 & 15 & 4.3 & 1.1 & \begin{tabular}{|l|l|}
0.88 \\
\end{tabular} & 28 \\
\hline 27 & 7.2 & 2.1 & 2.9 & $\begin{array}{ll}1.7 \\
\end{array}$ & 1.4 & 2.3 & $<0.02$ & 5.1 & 580 & 1.1 & $<0.05$ & 1.8 & 44 & 10 & 140 & 9 & 3.5 & 2.2 & 0.93 & 14 & 3.7 & 1 & 0.78 & 22 \\
\hline
\end{tabular}


Table 4 (continued). Summary of geochemical data for sediment samples (analyses by ICP-MS after acid digestion at USGS Denver)

\begin{tabular}{|c|c|c|c|c|c|c|c|c|c|c|c|c|c|c|c|c|c|c|c|c|c|c|}
\hline Sample & $\mathbf{L i}$ & Mn & Mo & Nd & $\mathbf{N i}$ & $\mathbf{P b}$ & Pr & $\mathbf{R b}$ & Sb & Se & Sm & Sn & $\mathbf{S r}$ & Tb & Th & TI & Tm & $\mathbf{U}$ & V & W & $\mathbf{Y b}$ & $\mathbf{Z n}$ \\
\hline & ppm & ppm & ppm & ppm & ppm & ppm & ppm & ppm & ppm & ppm & ppm & ppm & ppm & ppm & ppm & ppm & ppm & ppm & ppm & ppm & ppm & ppm \\
\hline A1A & 43 & 350 & 1.9 & 22 & 81 & 17 & 5.5 & 78 & 0.5 & 1 & 3.9 & 12 & 210 & 0.68 & 7.6 & 0.6 & 0.39 & 3.6 & 120 & 1.5 & 2.7 & 92 \\
\hline B1 & 43 & 370 & 1.6 & 22 & 79 & 13 & 5.6 & 78 & 0.4 & 1 & 4 & 14 & 220 & 0.7 & 7 & 0.5 & 0.36 & 3.5 & 120 & 1.3 & 2.7 & 94 \\
\hline $10 \mathrm{~A}$ & 29 & 300 & 0.7 & 15 & 57 & 8.5 & 3.8 & 72 & $<0.3$ & $<1$ & 3 & 4 & 190 & 0.5 & 4.6 & 0.4 & 0.26 & 1.8 & 90 & 0.7 & 1.8 & 76 \\
\hline 5 & 44 & 360 & 1.8 & 20 & 80 & 13 & 5.5 & 81 & 0.4 & 1 & 3.6 & 13 & 200 & 0.65 & 7.4 & 0.5 & 0.33 & 3.6 & 120 & 1.2 & 2.4 & 95 \\
\hline 6 & 40 & 360 & 2.3 & 20 & 80 & 13 & 4.9 & 74 & $<0.3$ & 1 & 3.8 & 12 & 240 & 0.65 & 6.9 & 0.5 & 0.32 & 3.3 & 120 & 1 & 2.3 & 93 \\
\hline 7 & 40 & 340 & 2.3 & 20 & 78 & 14 & 5.1 & 76 & $<0.3$ & $<1$ & 3.8 & 13 & 210 & 0.62 & 6.4 & 0.5 & 0.35 & 3.2 & 120 & 1.1 & 2.4 & 92 \\
\hline 8 & 44 & 360 & 1.6 & 20 & 82 & 13 & 5.2 & 80 & $<0.3$ & 1 & 3.7 & 9.5 & 200 & 0.64 & 7.1 & 0.5 & 0.36 & 3.4 & 120 & 1.2 & 2.6 & 99 \\
\hline 9 & 41 & 350 & 2.4 & 20 & 78 & 14 & 5.2 & 79 & 0.3 & 1 & 3.8 & 13 & 220 & 0.67 & 7.1 & 0.5 & 0.34 & 3.3 & 120 & 1.2 & 2.5 & 94 \\
\hline 11 & 43 & 360 & 1.8 & 21 & 82 & 12 & 5 & 75 & 0.3 & 1 & 3.7 & 13 & 200 & 0.66 & 6.5 & 0.5 & 0.35 & 2.9 & 120 & 1.1 & 2.6 & 96 \\
\hline 12 & 40 & 380 & 1.9 & 21 & 79 & 14 & 5.2 & 77 & $<0.3$ & 1 & 4 & 14 & 240 & 0.65 & 7.2 & 0.5 & 0.34 & 3 & 130 & 1.1 & 2.6 & 98 \\
\hline 13 & 42 & 380 & 2 & 18 & 80 & 13 & 4.9 & 75 & 1.3 & 1 & 3.4 & 31 & 220 & 0.63 & 6.8 & 0.5 & 0.33 & 3 & 120 & 1 & 2.3 & 91 \\
\hline 14 & 44 & 360 & 2.6 & 20 & 81 & 12 & 5.2 & 79 & 0.3 & 1 & 4 & 12 & 210 & 0.65 & 7.4 & 0.5 & 0.38 & 3.6 & 120 & 1.2 & 2.8 & 110 \\
\hline 15 & 25 & 240 & 0.5 & 13 & 45 & 7.9 & 3.2 & 80 & 0.3 & $<1$ & 2.6 & 3 & 190 & 0.38 & 3.9 & 0.3 & 0.22 & 1.6 & 81 & 0.4 & 1.4 & 79 \\
\hline 16 & 30 & 340 & 0.6 & 19 & 58 & 10 & 4.5 & 71 & 0.4 & $<1$ & 3.4 & 3 & 240 & 0.58 & 5.4 & 0.4 & 0.33 & 2.2 & 93 & 0.7 & 2.2 & 66 \\
\hline 17 & 36 & 380 & 0.7 & 20 & 67 & 10 & 5.1 & 83 & $<0.3$ & $<1$ & 3.7 & 2 & 240 & 0.64 & 7.4 & 0.4 & 0.36 & 2.4 & 110 & 0.8 & 2.4 & 77 \\
\hline 18 & 41 & 360 & 1.4 & 21 & 75 & 15 & 5.2 & 78 & $<0.3$ & $<1$ & 4 & 12 & 230 & 0.65 & 6.9 & 0.5 & 0.32 & 3.1 & 120 & 1.6 & 2.4 & 92 \\
\hline 19 & 20 & 440 & 0.6 & 26 & 44 & 12 & 6.1 & 48 & $<0.3$ & $<1$ & 4.5 & 2 & 270 & 0.7 & 9 & 0.4 & 0.33 & 2.4 & 96 & 0.8 & 2.3 & 44 \\
\hline 20 & 26 & 380 & 0.6 & 17 & 43 & 9.4 & 4.4 & 55 & $<0.3$ & $<1$ & 3.4 & 4 & 260 & 0.6 & 8.9 & 0.5 & 0.29 & 2.5 & 84 & 0.6 & 2.2 & 53 \\
\hline 21 & 25 & 360 & 0.6 & 20 & 48 & 10 & 4.7 & 54 & $<0.3$ & $<1$ & 3.5 & 2 & 240 & 0.58 & 5.5 & 0.5 & 0.33 & 2 & 84 & 0.6 & 2.3 & 55 \\
\hline 22 & 8.4 & 140 & 0.2 & 21 & 2 & 6.3 & 5.7 & 34 & $<0.3$ & $<1$ & 2.9 & 0.8 & 1100 & 0.38 & 5.8 & 0.3 & 0.16 & 0.77 & 24 & $<0.1$ & 0.98 & 20 \\
\hline 23 & 4.1 & 91 & 0.1 & 6.8 & $<0.1$ & 4.7 & 1.7 & 11 & $<0.3$ & $<1$ & 0.99 & $<0.5$ & 2300 & 0.16 & 1.1 & $<0.1$ & 0.068 & 0.63 & 13 & $<0.1$ & 0.5 & 7 \\
\hline 24 & 27 & 300 & 0.5 & 17 & 41 & 8.9 & 4.2 & 56 & $<0.3$ & $<1$ & 3.1 & 2 & 260 & 0.53 & 4.7 & 0.6 & 0.26 & 1.9 & 83 & 0.5 & 1.9 & 55 \\
\hline 25 & 28 & 440 & 0.7 & 20 & 52 & 9.4 & 4.7 & 64 & $<0.3$ & $<1$ & 3.6 & 2 & 280 & 0.66 & 6.3 & 0.5 & 0.3 & 2 & 99 & 1.1 & 2.4 & 58 \\
\hline 26 & 24 & 450 & 0.6 & 25 & 48 & 11 & 6.1 & 62 & $<0.3$ & $<1$ & 4.5 & 2 & 280 & 0.68 & 6.7 & 0.5 & 0.34 & 2.1 & 93 & 0.6 & 2.4 & 55 \\
\hline 27 & 26 & 380 & 0.5 & 20 & 50 & 9.7 & 4.8 & 54 & $<0.3$ & $<1$ & 3.7 & 2 & 260 & 0.6 & 5.5 & 0.4 & 0.32 & 1.8 & 90 & 0.6 & 2.2 & 51 \\
\hline & & & & & & & & & & & & & & & & & & & & & & \\
\hline
\end{tabular}




\section{Results and discussion}

\section{Seabed gamma spectrometry}

The EEL data were converted in real time into approximate sea floor concentrations of radionuclides. This was done by comparing each 5-s gamma-ray spectrum to standard spectra for $\mathrm{K}, \mathrm{U}, \mathrm{Th}$, and Cs (Hendriks et al., 2001). The standard spectra were obtained from measurements made with the detector in a tank of water on each of a set of calibration pads. The K, $\mathrm{U}$, and Th pads are concrete blocks containing known amounts of $\mathrm{K}, \mathrm{U}$, and Th. A fourth, background, pad is a plain concrete block with no added radioactive material. The Cs pad consists of a wooden box containing fine sand from the Irish Sea contaminated by ${ }^{137} \mathrm{Cs}$ from the authorised discharges of the Sellafield nuclear plant.

Examination of the EEL data during the survey suggested only very low seabed levels of gammaemitting artificial radionuclides such as ${ }^{137} \mathrm{Cs}$; no obvious ${ }^{137} \mathrm{Cs}$ peaks were noticed in the sea bed gammaray spectra (e.g., fig. 8). The variations seen in sea floor radioactivity were in natural isotopes (of $\mathrm{K}$ and the $\mathrm{U}$ and Th decay series) attributable to geological features. These are most obvious at the $90-\mathrm{m}$ site where features seen on sidescan sonar records (fig. 9) have a distinctive response in the radiometric and other EEL data (fig. 10).

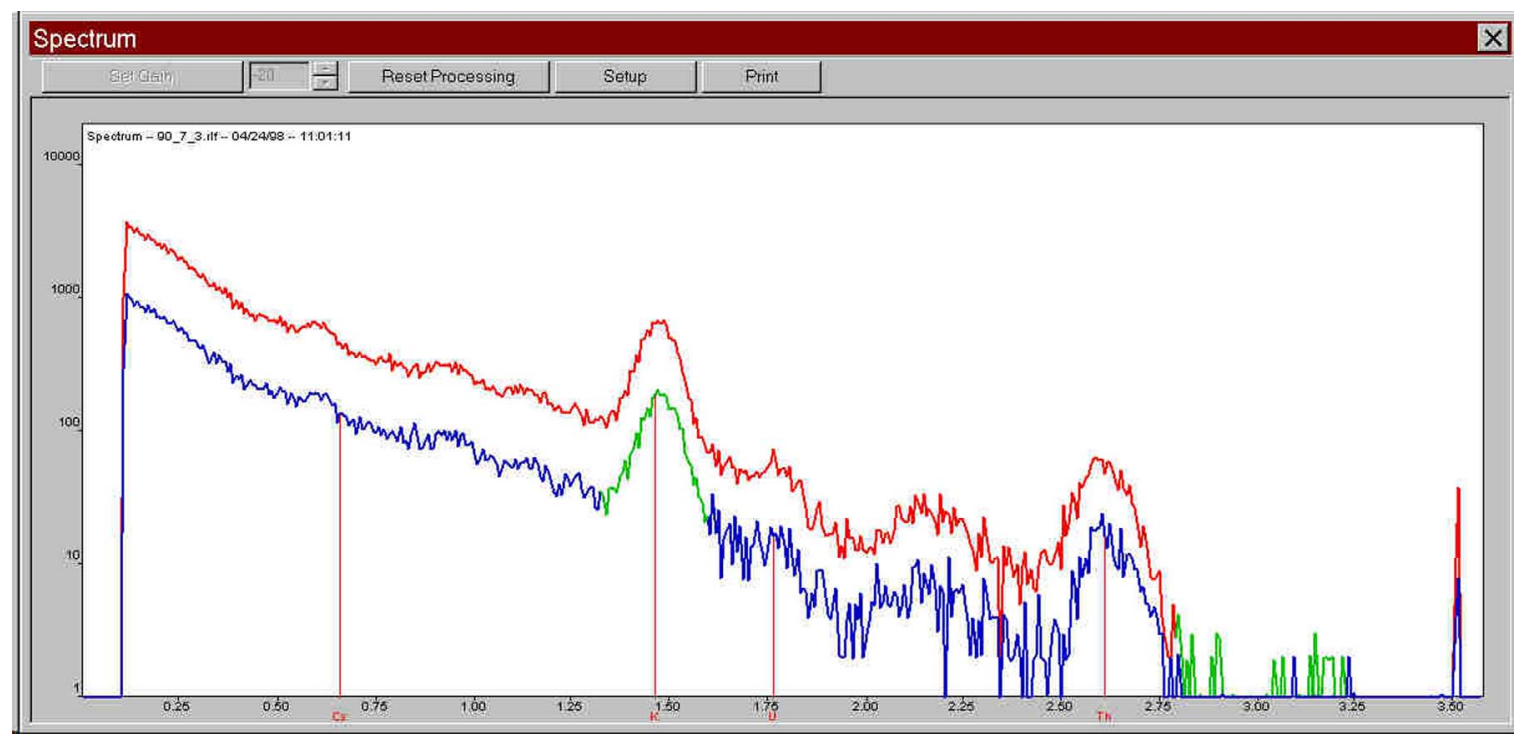

Figure 8. Typical seabed gamma-ray spectrum from the survey. Horizontal axis = gamma-ray energy $(\mathrm{MeV})$, vertical axis $=$ counts. The red vertical lines indicate the positions of the ${ }^{137} \mathrm{Cs}(0.66 \mathrm{MeV}),{ }^{40} \mathrm{~K}$ $(1.46 \mathrm{MeV}),{ }^{214} \mathrm{Bi}(\mathrm{U})(1.76 \mathrm{MeV})$, and ${ }^{208} \mathrm{Tl}(\mathrm{Th})(2.62 \mathrm{MeV})$ lines

Line 90_3 (fig 10a) shows a generally uniform total gamma signal over a smooth sediment-covered sea floor. Two small upstanding features on the seabed (seen on the pressure trace and as high noise features) correspond to gravel waves seen clearly on the sidescan mosaic (fig. 9). These do not show any major difference in total count compared to the surrounding finer grained sediments.

In contrast, the rough areas of sea floor on Line 90_7 (fig. 10b), which are apparent on the sound and pressure traces, have a markedly higher total count signal than their surroundings. These features match rock outcrops seen on the sidescan image (fig. 9) and are probably (from their radiometric signature) granitic, an interpretation consistent with their proximity to the granite ridge of the Farallon Islands (NOAA, 1990). Line 90_7 also shows a change in sediment type along its length. The left hand end of the 


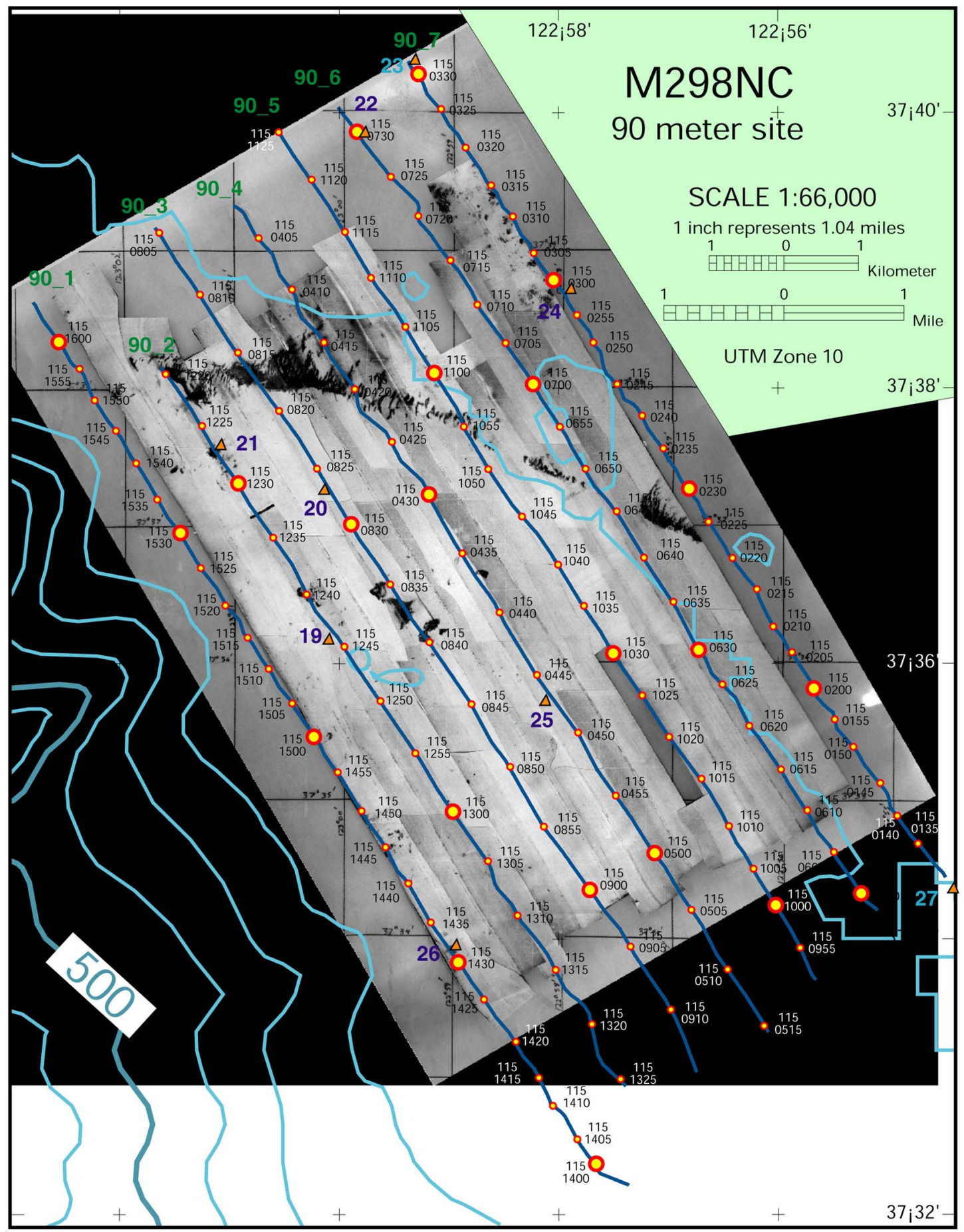

Figure 9. Sidescan mosaic for 90-m area.

segment of line depicted in figure 10b shows a general total count base level of about 100 counts per second between the outcrops. This is consistent with siliciclastic sediments. In contrast, the right hand end of the line shows sediments with a total count of only around 50 counts per second. These were interpreted as shelly sands, and this was confirmed by sampling. 


\section{Line 90_3}

Sound \% Gamma cps

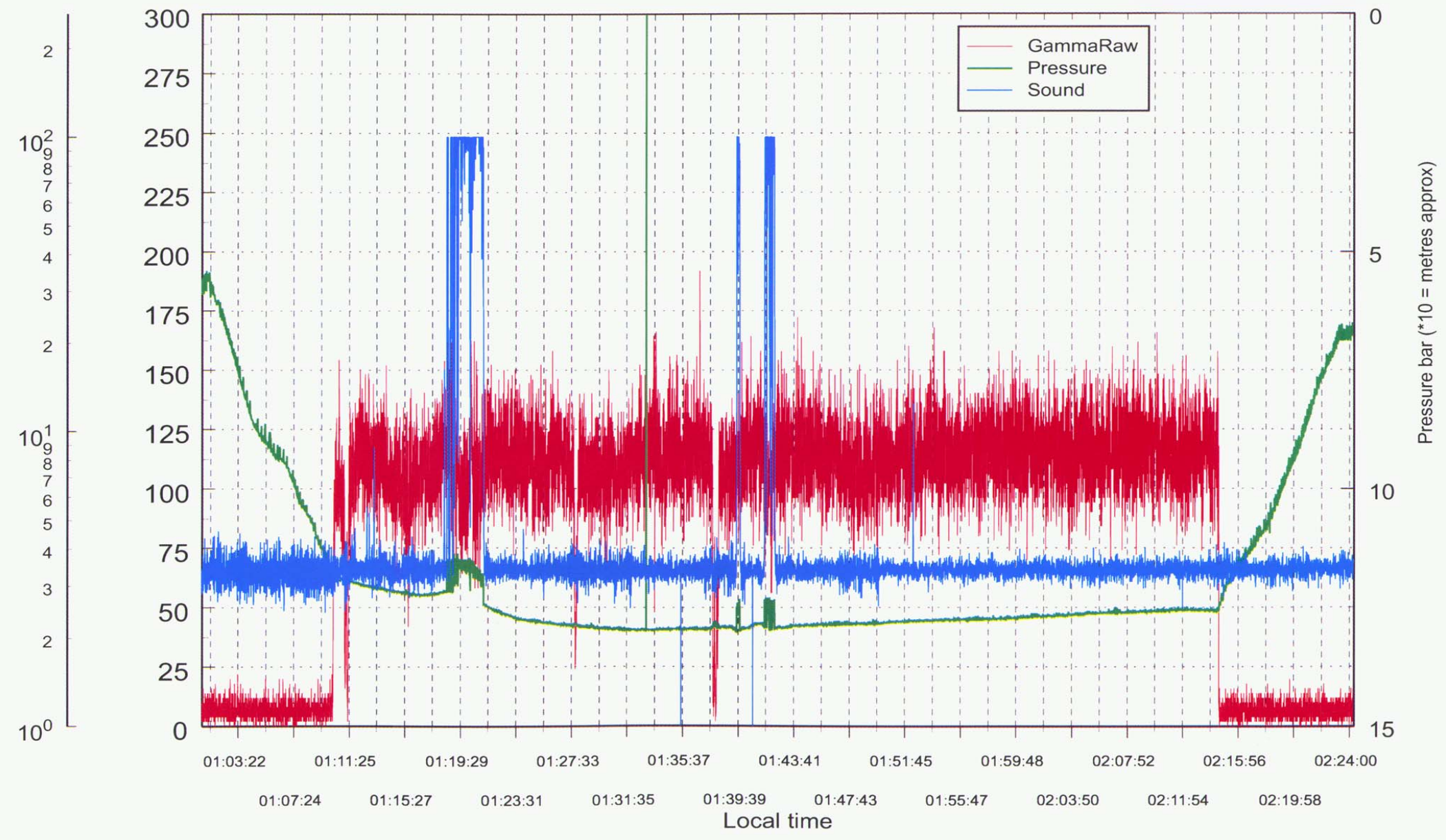

Figure. 10A. Examples of geological variation of gamma spectrometer data. Line 90_3 (complete line). 


\section{Line 90_7}

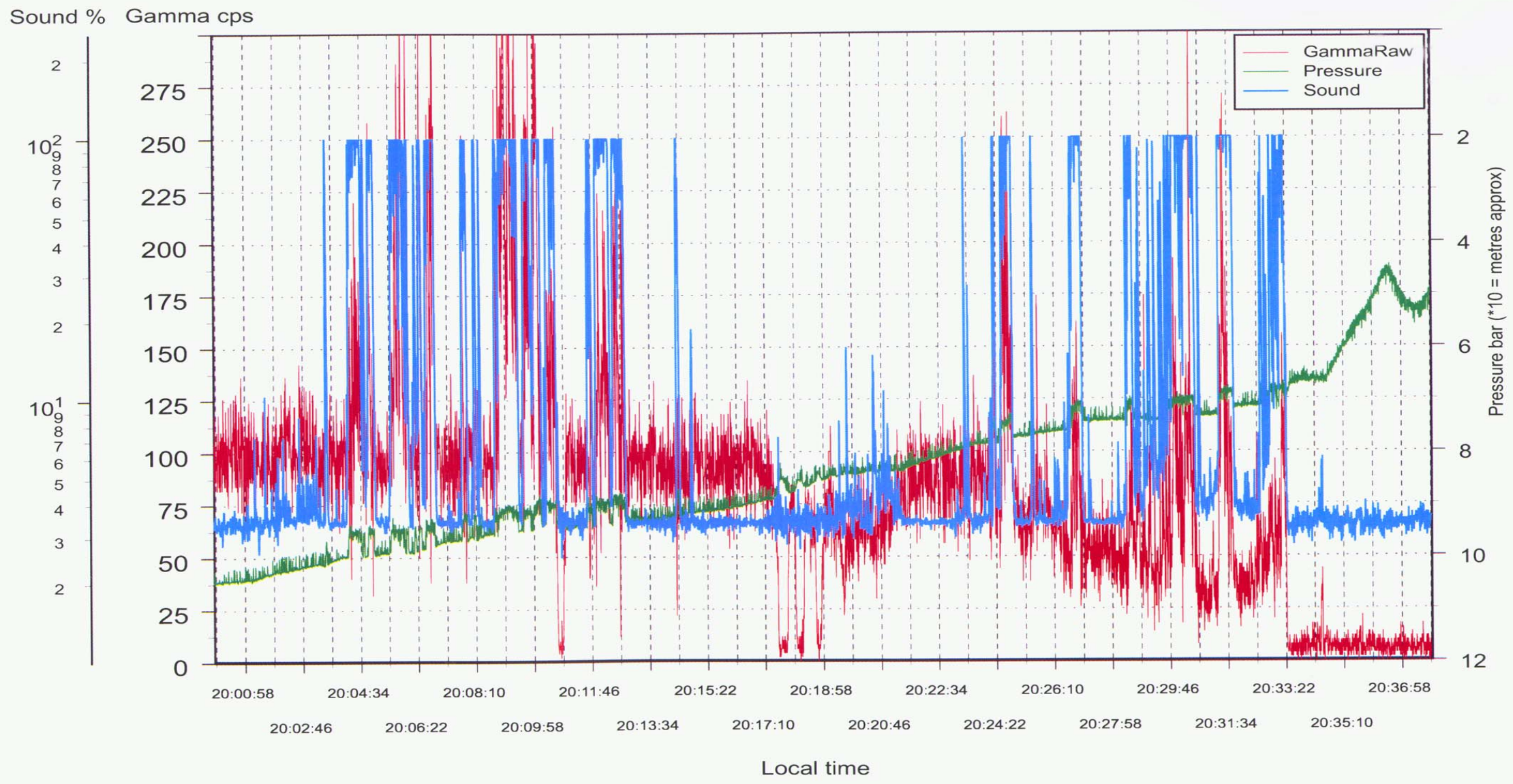

Figure. 10B. Examples of geological variation of gamma spectrometer data. Line 90_7 (N end of line approximately $\mathrm{N}$ of sample site 24, fig. 10) . 
After the survey, the gamma-ray spectra were postprocessed to optimise the sea floor data for individual radionuclides. Particular attention was given to the ${ }^{137} \mathrm{Cs}$ data. Initially this was done for each 5-s gamma-ray spectrum, but this produced very noisy Cs results owing to the very low levels present. A second stage of the postprocessing was therefore undertaken. This involved integrating the data over longer periods of time to try to obtain more reliable ${ }^{137} \mathrm{Cs}$ values. Data were compared for one test line (900_7) on the basis of spectra integrated over 5 -s, 50 -s, 150 -s and 500 -s periods (corresponding to approximately 5 , 50,150 , and $500 \mathrm{~m}$ of seabed towed). When the integration period was extended to $500 \mathrm{~s}$, variations along the test line showed a coherent structure and the highest values occurred where the line came closest to the known highest concentrations of barrels on the sea floor (fig. 11). This suggested that meaningful data might be extracted over 500 -s periods.

The postprocessing was therefore re-run using 500-s integration, on the basis of moving averages along each line, to assess whether there was a geographical coherence to the ${ }^{137} \mathrm{Cs}$ values. One data file had become corrupted (line 900_6) and was not available for postprocessing.

The 500-s data for the 900-m site data appear to show a coherent pattern, with the connection of relatively high and low values between survey lines (fig. 12). This suggests some confidence can be placed in the data. However, the levels of ${ }^{137} \mathrm{Cs}$ present must be regarded as being close to the detection limit of the system, even for 500 -s counting periods, and the data should be treated with caution.

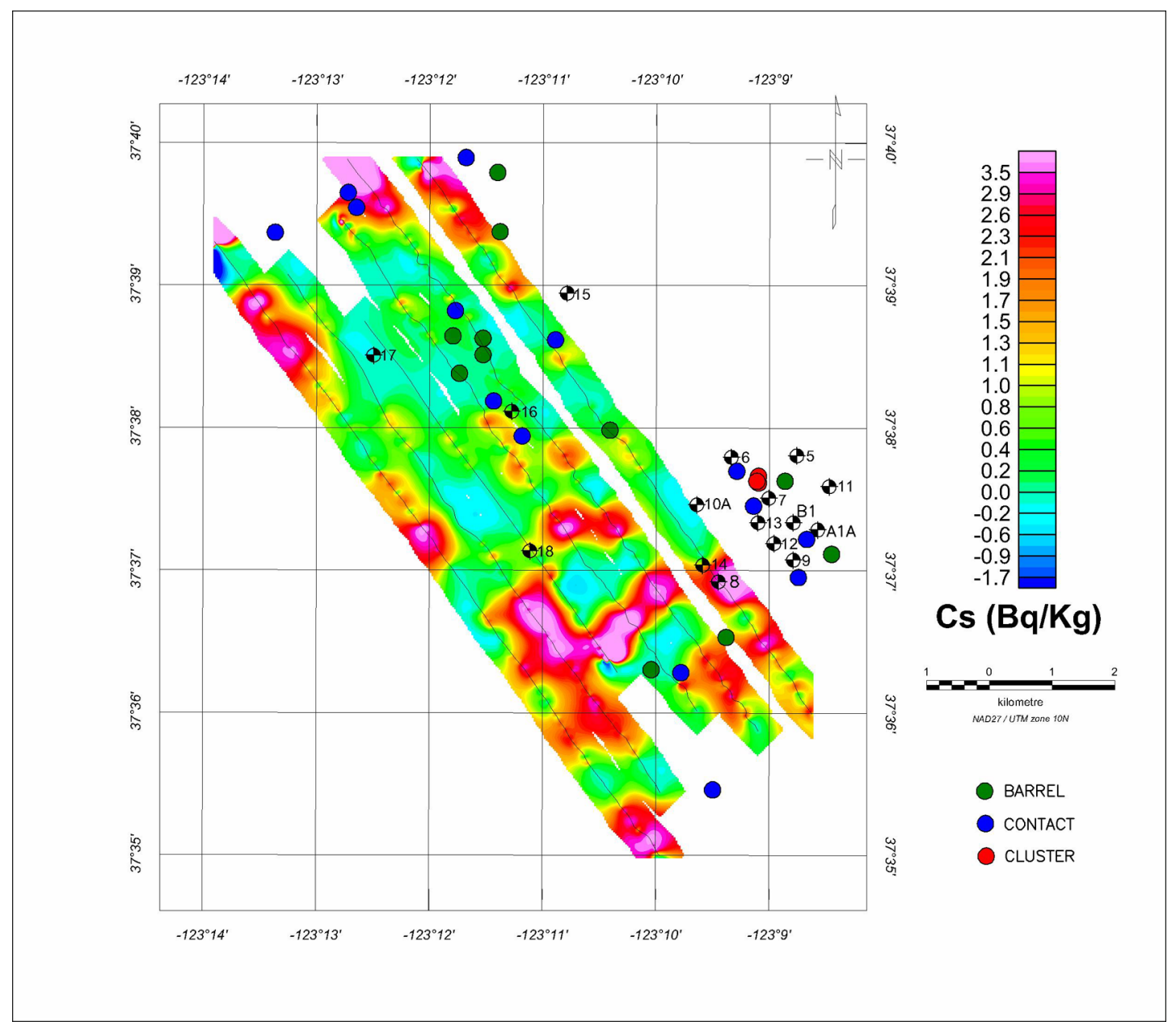

Figure 12. ${ }^{137} \mathrm{Cs}$ data for $900-\mathrm{m}$ site. Typical uncertainties $(1 \sigma)$ are about 15 percent. 


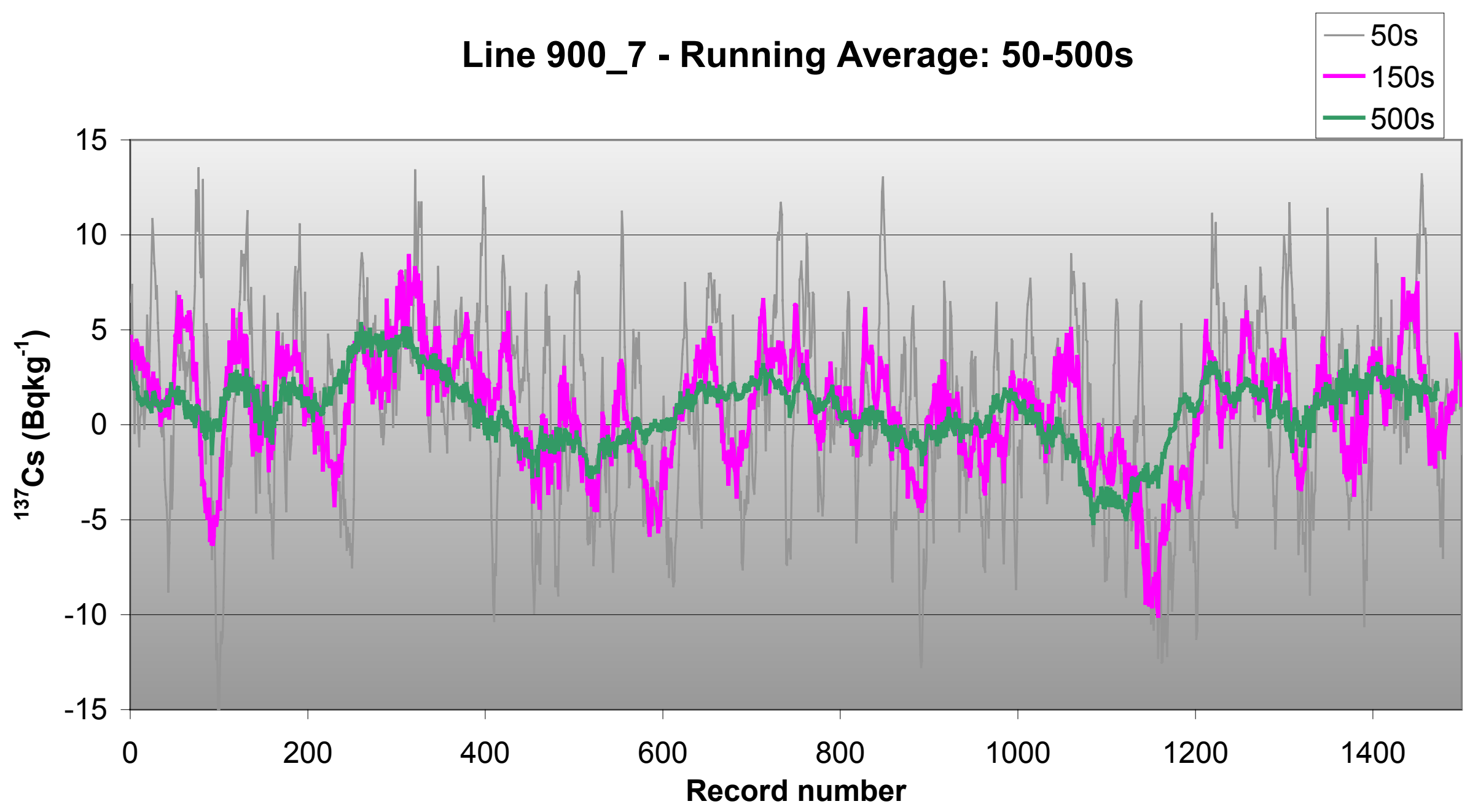

Figure 11. ${ }^{137} \mathrm{Cs}$ data integrated over 5, 50, 150 and $500 \mathrm{~s}$. Each record number represents one 5 s count covering about $5 \mathrm{~m}$ of seabed. 
A background was removed, initially, from the full postprocessed dataset. This was designed to remove the effects of seawater and the detector itself. Seawater contains some $50 \mathrm{~Bq} / 1$ of ${ }^{40} \mathrm{~K}$, and the detector has a small amount of ${ }^{207} \mathrm{Bi}$, which mainly affects the $\mathrm{U}$ and ${ }^{137} \mathrm{Cs}$ data (because of the proximity of the $570 \mathrm{keV}^{207} \mathrm{Bi}$ peak to the $662 \mathrm{keV}^{137} \mathrm{Cs}$ peak and the $609 \mathrm{keV}{ }^{214} \mathrm{Bi}$ peak). However, background removal caused a relative increase in the Cs values, compared with those obtained with no background subtraction. The ${ }^{137} \mathrm{Cs}$ concentrations from nonbackground corrected data are closer to those obtained by sample analysis (table 1). Also, the statistical fit of the spectra with the standard spectra is, paradoxically, better when no background is used. This may reflect the different operating conditions under which the standard spectra had to be collected under, owing to lack of time, compared with the background data. The latter were gathered under survey conditions, whereas the standard spectra were obtained using the same detector, but a different cable and electronics.

As the background correction largely causes a uniform shift in the final ${ }^{137} \mathrm{Cs}$ and other radionuclide concentrations, it does not affect significantly the relative values. In order to keep the ${ }^{137} \mathrm{Cs}$ figures (and those for other radionuclides) closer to the more accurate sample analyses, the data are presented without background removal (figs. 12-23).

The postprocessed seabed data show some correspondence between higher seabed ${ }^{137} \mathrm{Cs}$ concentrations and proximity to the known locations of barrels at the 900-m and cluster sites (figs. 12 and 13). There are also relatively high values where no barrels have been located. This might be expected to occur where barrels are too degraded to produce a detectable sidescan response. It should be noted that the total number of targets identified using sidescan data represents only a small proportion of the total number of barrels reportedly disposed of at the 900-m site.

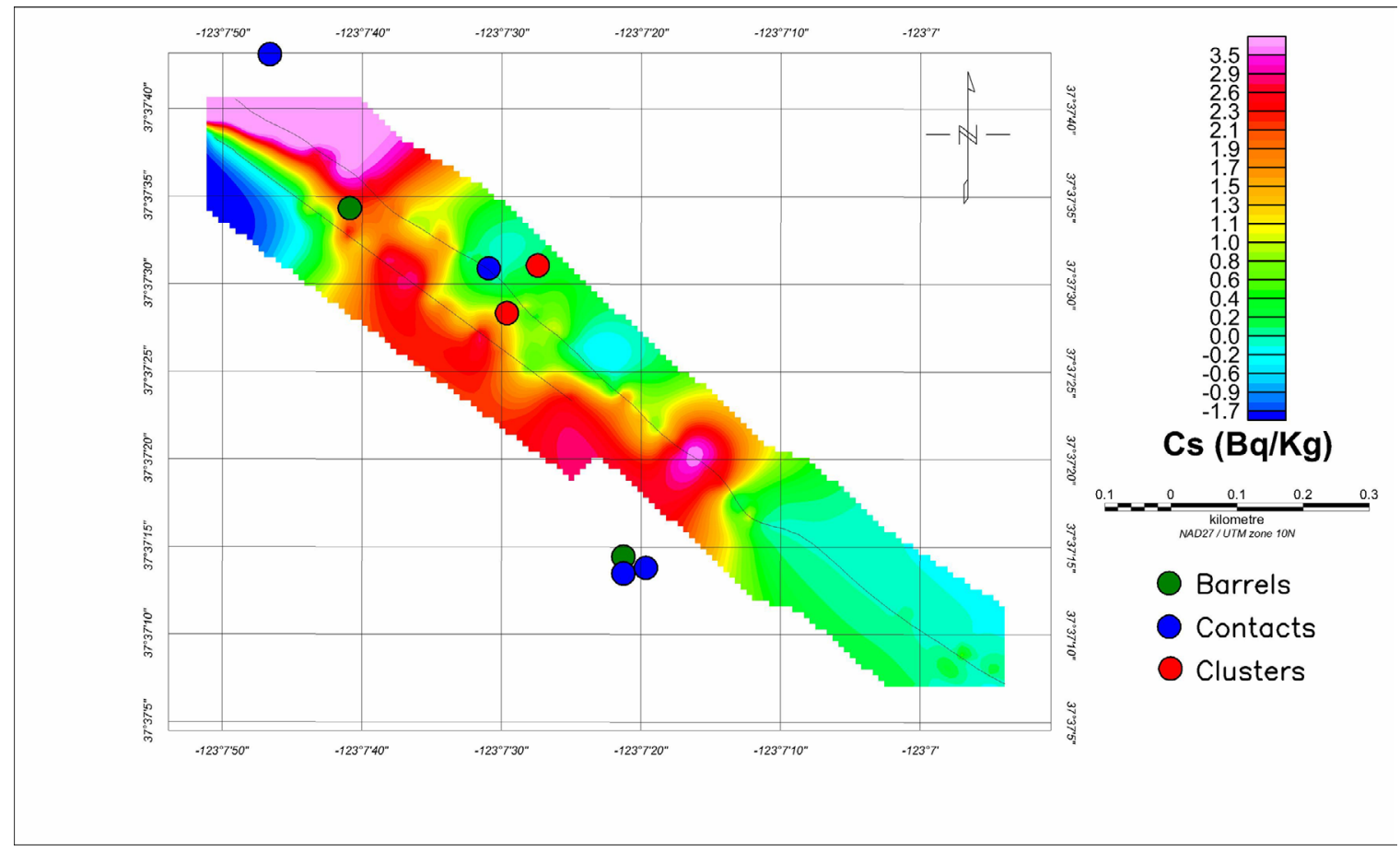

Figure 13. ${ }^{137} \mathrm{Cs}$ data for Cluster site. Typical uncertainties $(1 \sigma)$ about 15 percent.

There are also relatively low ${ }^{137} \mathrm{Cs}$ levels around some barrel clusters. This again is to be expected; the barrels could be intact, or at least have not suffered leakage, or there may not be any Cs present in those barrels. 
Overall, the data indicate very low ${ }^{137} \mathrm{Cs}$ contents across the survey area. There are no suggestions of any significant regional scale contamination of the sediments by ${ }^{137} \mathrm{Cs}$, nor of any localised concentrations of this radionuclide.

The postprocessed $500-\mathrm{s}{ }^{137} \mathrm{Cs}$ data for the $90 \mathrm{~m}$ site are also apparently quite coherent. The $90-\mathrm{m}$ ${ }^{137} \mathrm{Cs}$ values are lower overall than the $900 \mathrm{~m}$ and cluster data (figs. 12-14), which fits with the lower concentrations of ${ }^{137} \mathrm{Cs}$ observed in sample analyses (table 1). Similarly low levels were seen on the two 1,800 -m lines (fig. 15). Higher levels of ${ }^{137} \mathrm{Cs}$ at the 90 -m site appear to be very localised. There is no real correlation between the Cs data at the $90-\mathrm{m}$ site and those for $\mathrm{K}, \mathrm{U}$, and Th, making the data difficult to interpret in terms of seabed geology (figs. 14 and 16-19).



Figure 14. ${ }^{137} \mathrm{Cs}$ data for $90-\mathrm{m}$ site. Typical uncertainties $(1 \sigma)$ about 15 percent

\section{Laboratory analysis}

Subsequent laboratory sample analysis has confirmed the low levels of ${ }^{137} \mathrm{Cs}\left(<5 \mathrm{Bqkg}^{-1}\right)$ (table 1). The data are consistent with concentrations less than $10 \mathrm{Bqkg}^{-1}{ }^{137} \mathrm{Cs}$ reported from earlier studies (Dyer, 1976; NOAA, 1990; Noshkin et al., 1978; Schell and Sugai, 1980). Gamma-ray spectrometry indicated that similarly low levels of ${ }^{241} \mathrm{Am}$ may be present in a few samples. This was confirmed by alpha spectrometry of a selected number of samples (table 2). The latter analysis reveals detectable ${ }^{241} \mathrm{Am}$, in four of the eight samples selected, at levels below $2 \mathrm{Bqkg}^{-1}$. Plutonium isotopes were also detected; ${ }^{239,240} \mathrm{Pu}$ in all eight 


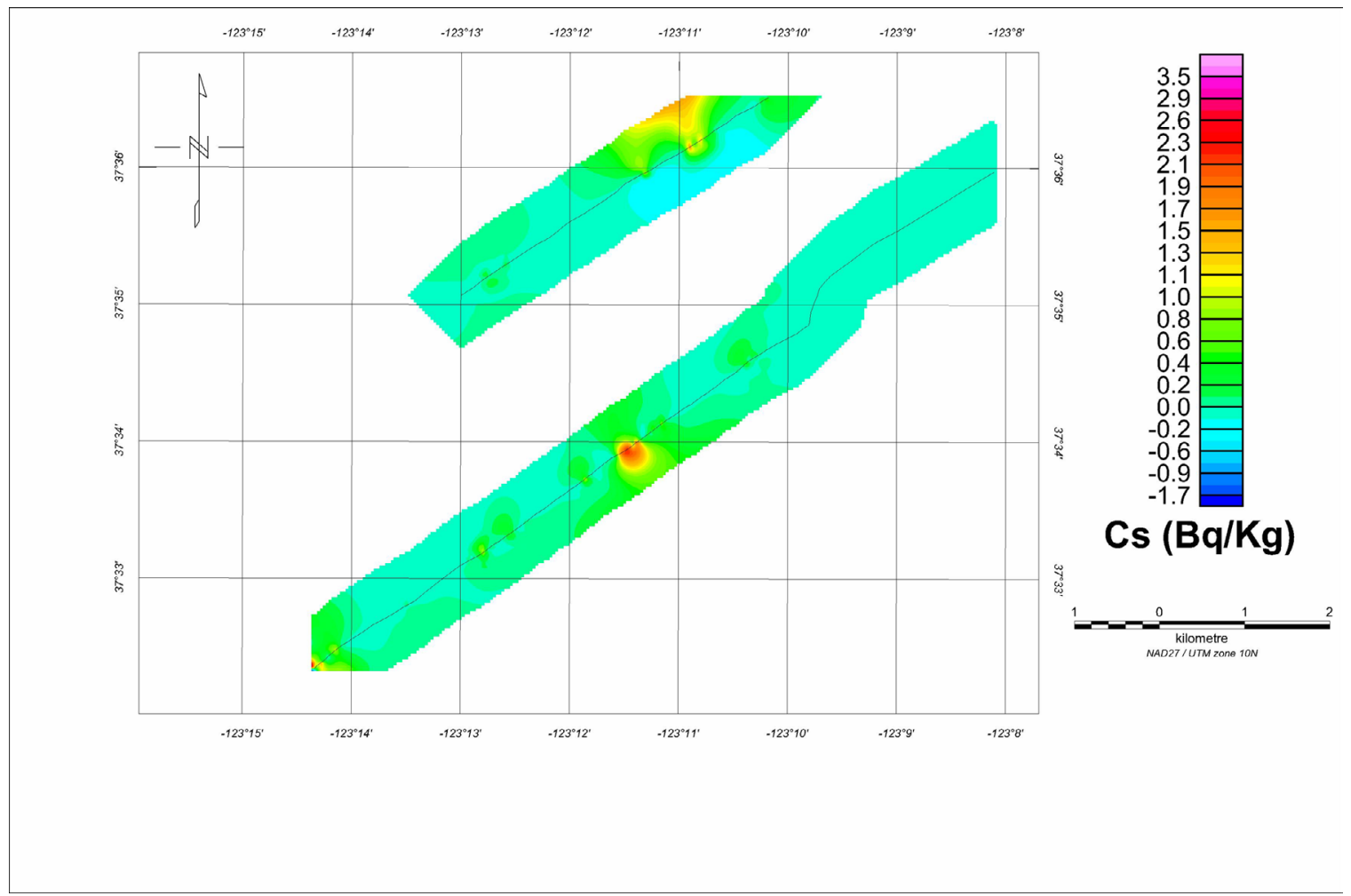

Figure 15. ${ }^{137} \mathrm{Cs}$ data for $1800-\mathrm{m}$ site. Typical uncertainties $(1 \sigma)$ about 15 percent.

samples at similarly low levels to ${ }^{241} \mathrm{Am}$ (no greater than $3 \mathrm{Bqkg}^{-1}$ ) and ${ }^{238} \mathrm{Pu}$ only in sample 5 . Again, these results are in line with previous work with ${ }^{239+240} \mathrm{Pu}$ concentrations up to $5 \mathrm{Bqkg}^{-1}$ being reported (Noshkin et al., 1978; Schell and Sugai, 1980), whilst Dyer (1976) found slightly higher levels, in some samples, up to $20 \mathrm{Bqkg}^{-1}$ and ${ }^{238} \mathrm{Pu}$ contents of up to $5 \mathrm{Bqkg}^{-1}$.

These concentrations are well below the natural background levels of ${ }^{40} \mathrm{~K}$ (typically $400-600$ $\left.\mathrm{Bqkg}^{-1}\right)$ and generally below the concentrations of $\mathrm{U}\left({ }^{214} \mathrm{Bi}\right)$ and $\mathrm{Th}\left({ }^{208} \mathrm{Tl}\right)$, which mostly range from 15-50 and 15-30 $\mathrm{Bqkg}^{-1}$ respectively. Whether the sediments are contaminated in excess of what would be expected from nuclear weapons fallout has been a matter of some controversy (NOAA, 1990). What is clear is that the concentrations of ${ }^{137} \mathrm{Cs},{ }^{241} \mathrm{Am}$, and $\mathrm{Pu}$ isotopes are considerably lower than levels encountered in Irish Sea sediments (e.g., Jones et al., 1999; Kershaw et al., 1999). These reach maxima 2 or 3 orders of magnitude higher than those observed from the Farallons, but they do not give rise to doses in excess of annual limits, either through direct exposure or through the food chain (e.g. FSA/SEPA, 2000).

${ }^{137} \mathrm{Cs}$ was detected in all the 90-m samples except for two with very high shell contents (Nos. 22 and 23). Three of the $900-\mathrm{m}$ site samples had no detectable ${ }^{137} \mathrm{Cs}$. Slightly higher (albeit still very low) levels of ${ }^{137} \mathrm{Cs}$ occur in samples taken near known barrels, and some of these have detectable ${ }^{241} \mathrm{Am}$. Away from the 'cluster' locations, ${ }^{241} \mathrm{Am}$ was only detected in one sample (from the 90-m site) at an extremely low level $\left(0.2 \mathrm{Bqkg}^{-1}\right)$ by $\alpha$ spectrometry. Similarly, Pu contents of the sediment samples are higher in the vicinity of known barrels than elsewhere in the 900-m site or the 90-m site. The differences in Cs, Am, and $\mathrm{Pu}$ levels between the 'cluster' samples and other locations could be due to inputs of radioactivity from the waste in the barrels or the result of differential input/uptake of fallout radionuclides.

Barrel leakage is indicated by the coincidence of higher values of $\mathrm{Cs}, \mathrm{Am}$, and Pu with the barrel clusters and the generally similar levels of natural radioactivity in the samples, regardless of Cs and Am content. It is further supported by the poor condition of at least some barrels. There have been differing interpretations made of previous sample analyses, but most authors conclude that there has been some input 


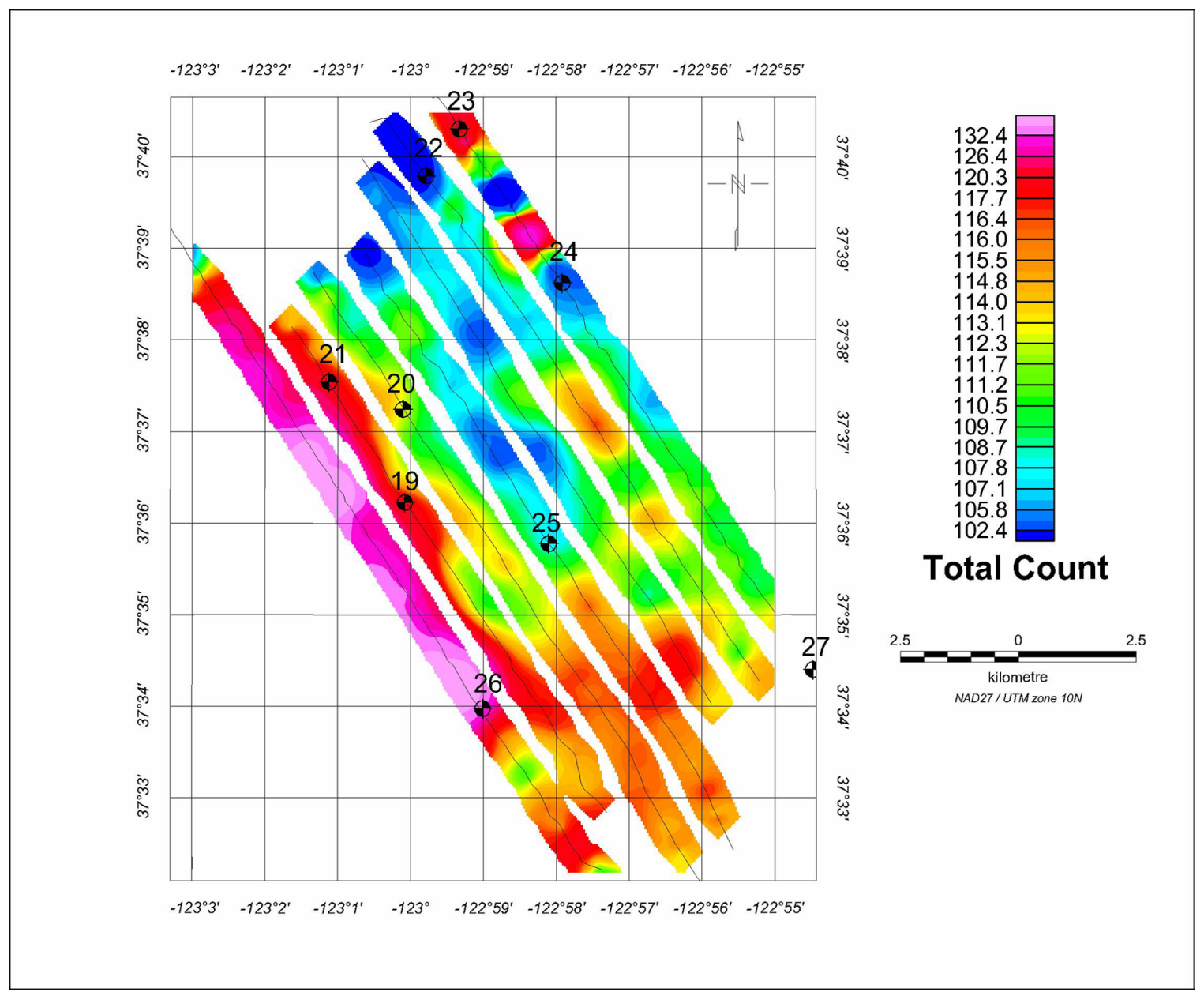

Figure 16. Total count data for $90-\mathrm{m}$ site $\left(\right.$ counts. $\left.\mathrm{s}^{-1}\right)$. Typical uncertainties $(1 \sigma)$ better than 1 percent.

to the sediments from the barrels (NOAA, 1990; Schell and Sugai, 1980). If barrel leakage has occurred, then there are no indications, in the present data, of any significant enhancement of radionuclide levels on a regional scale in the areas surveyed.

Alternative explanations are that the variations are due to differences in fallout radionuclide input or uptake. The latter could be influenced by differences in sedimentation rate, with fallout particles being more efficiently scavenged from the water column or, conversely, diluted by higher rates of sediment buildup.

Sample data do indicate clear differences between the shallow water sediments at the 90-m site and those from the much deeper $900-\mathrm{m}$ site (fig. 24; tables 1-4). The 900-m site has finer grained sediments (figs. 25 and 26); mud contents at the 90 -m site are less than $40 \%$ with clay contents below about $10 \%$, whereas mud contents at the $900-\mathrm{m}$ site are mostly above $30 \%$, ranging up to over $90 \%$, and clay contents are in the range of $10-40 \%$. The main geochemical differences between the two areas lie in higher Fe and $\mathrm{Mg}$ values at the $900-\mathrm{m}$ site, along with a range of trace elements, including $\mathrm{As}, \mathrm{Cr}, \mathrm{Cu}, \mathrm{Ge}, \mathrm{Li}, \mathrm{Mo}, \mathrm{Ni}, \mathrm{Pb}$, $\mathrm{Rb}, \mathrm{Sn}, \mathrm{U}, \mathrm{V}, \mathrm{W}$, and $\mathrm{Zn}$ (Fig. 24; Table 4). Levels of $\mathrm{Al}$ and $\mathrm{K}$ are broadly comparable. This suggests a different mix of clay minerals at the sites with $\mathrm{Fe}$ and Mg-rich species (e.g., smectite), with a great affinity for many trace elements, being more important in deeper water. An increase in smectite with increasing water depth has been noted in mineralogical studies of the area (Booth et al., 1989; Griggs and Hein, 1980). 


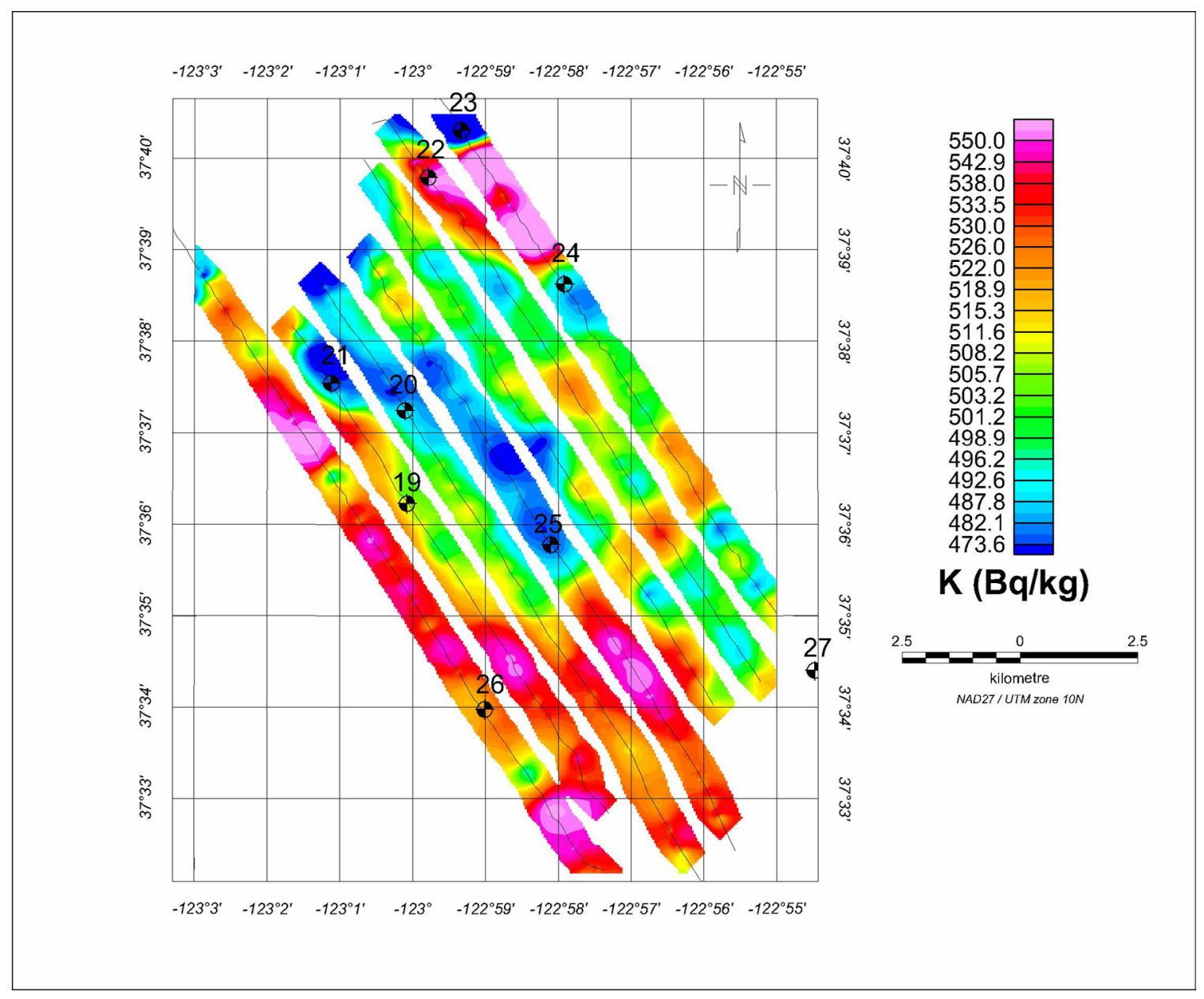

Figure 17. $\mathrm{K}$ data for the 90 -m site. Typical uncertainties $(1 \sigma)$ better than 2 percent.

There is, however, no obvious relationship between ${ }^{137} \mathrm{Cs}$ content and the grain size of the sediments (Figs. 25 and 26), or their geochemistry. Thus the data do not support the preferential uptake of ${ }^{137} \mathrm{Cs}$ in the 'cluster' area owing to an increased sorption capacity for Cs of the sediments.

Geochemicallyand texturally the 'cluster' site is very similar to the remainder of the $900-\mathrm{m}$ site (fig. 24; tables 3 and 4). This suggests that the slightly higher contents of radionuclides in the vicinity of known barrels are most likely due to leakage from the barrels. However, the differences in radionuclide contents between the 90-m and 900-m sites could in part be due to the increased sorption capacity of the deeper water, relatively smectite-rich sediments. An increase in radionuclide retention capacity of the deeper sediments was suggested by the data of Booth et al. (1989).

$\mathrm{U}$ and Th concentrations in the bottom sediments are all well within normal ranges for sediments (Wedepohl, 1978) in both the EEL and the sample data (figs. 18, 19, 22-24 and tables 1 and 4). Levels of U are higher in the deeper water sediments, reflecting the greater proportion of finer material, but Th values are broadly similar across the whole survey area. As for Cs, there is no clear-cut relationship between $\mathrm{U}$ or Th content and known barrel locations. Some higher Th and/or U areas coincide with barrels, but other barrel locations are associated with lower Th and/or U. Since the levels of $U$ and Th do not differ from natural background values, there is no need to invoke an input from the barrels to explain the distribution. However, the generally higher contents of $\mathrm{U}$ and $\mathrm{Th}$ in the sediments from the cluster site, when compared with elsewhere in the 900-m site, may indicate a slight enhancement due to input from the barrels. U and Th 


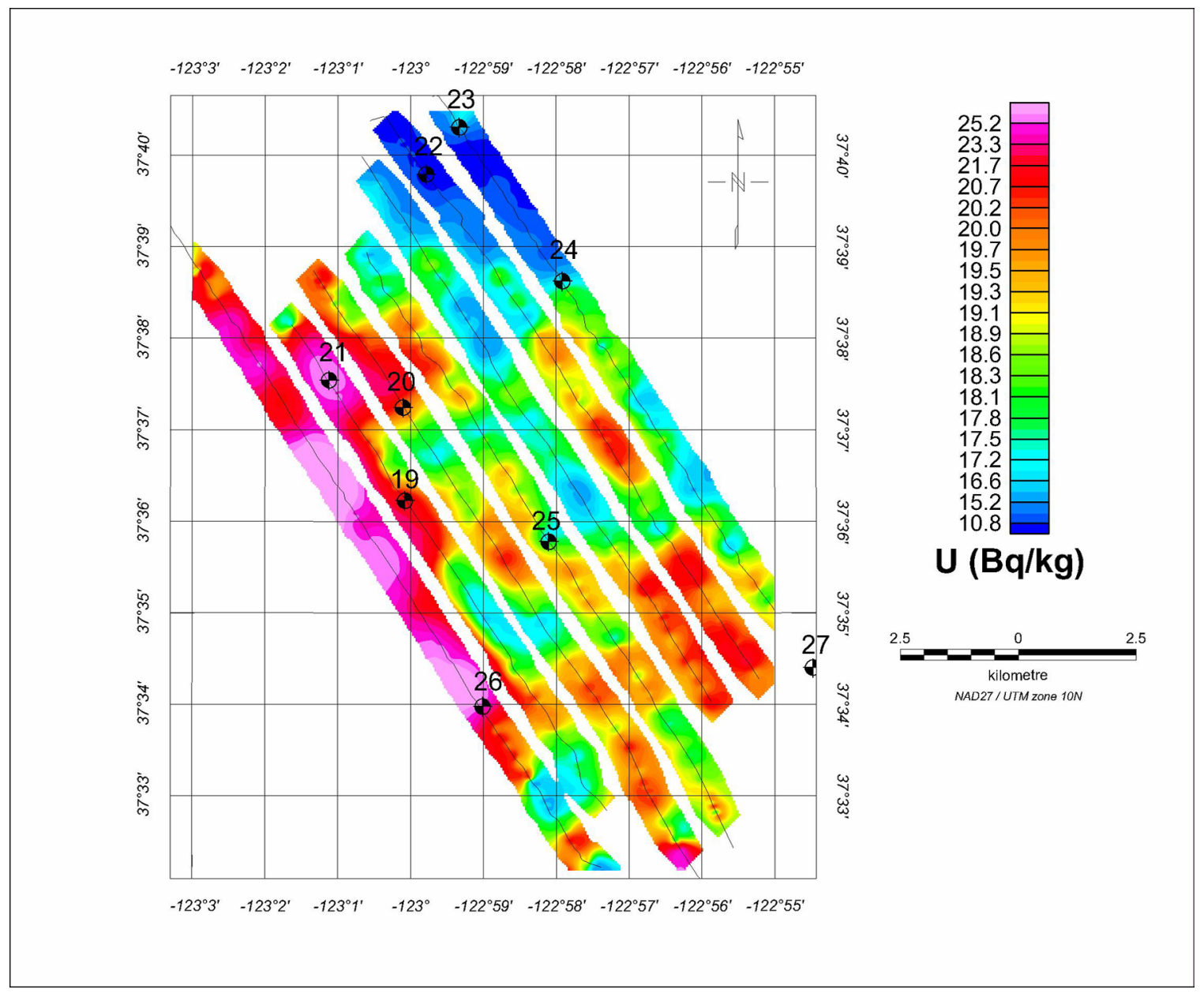

Figure 18. U data for the $90-\mathrm{m}$ site. Typical uncertainties $(1 \sigma)$ better than 25 percent.

are listed as being present in the wastes disposed at the Farallon Islands Radioactive Waste Dump (NOAA, 1990; Noshkin et al., 1978).

Geochemical analyses of the sediment samples do not indicate any significant enhancement of other contaminants such as heavy metals or Be (fig. 24; table 4). Sn is the only element reported at levels that are appreciably above those expected for average fine-grained sediments or rocks (Turekian and Wedepohl, 1961). Concentrations of Sn are approximately twice the average shale levels (of $6 \mathrm{ppm}$ ) in most samples from the cluster site, and one from the $900-\mathrm{m}$ site, with a maximum of $31 \mathrm{ppm}$ in sample 13. Since $\mathrm{Hg}$ and organic contaminants were not measured in the samples, it is not possible to comment on the impact, if any, of the disposal of other types of waste in the area.

\section{Conclusions}

Both in situ measurements and laboratory analyses of sediment samples indicate only very low levels of artificial radionuclides in the surveyed areas of the Farallon Dump Site. Concentrations of ${ }^{137} \mathrm{Cs}$, ${ }^{241} \mathrm{Am},{ }^{239+240} \mathrm{Pu}$ and ${ }^{238} \mathrm{Pu}$, in the sediments are all below $10 \mathrm{Bqkg}^{-1}$. These are significantly lower than natural background levels of $\mathrm{K}, \mathrm{U}$, and Th. They are also well below concentrations encountered in Irish Sea sediments, which arise from authorised discharges of waste from the Sellafield nuclear reprocessing plant. There appears to be some indication of leakage of 


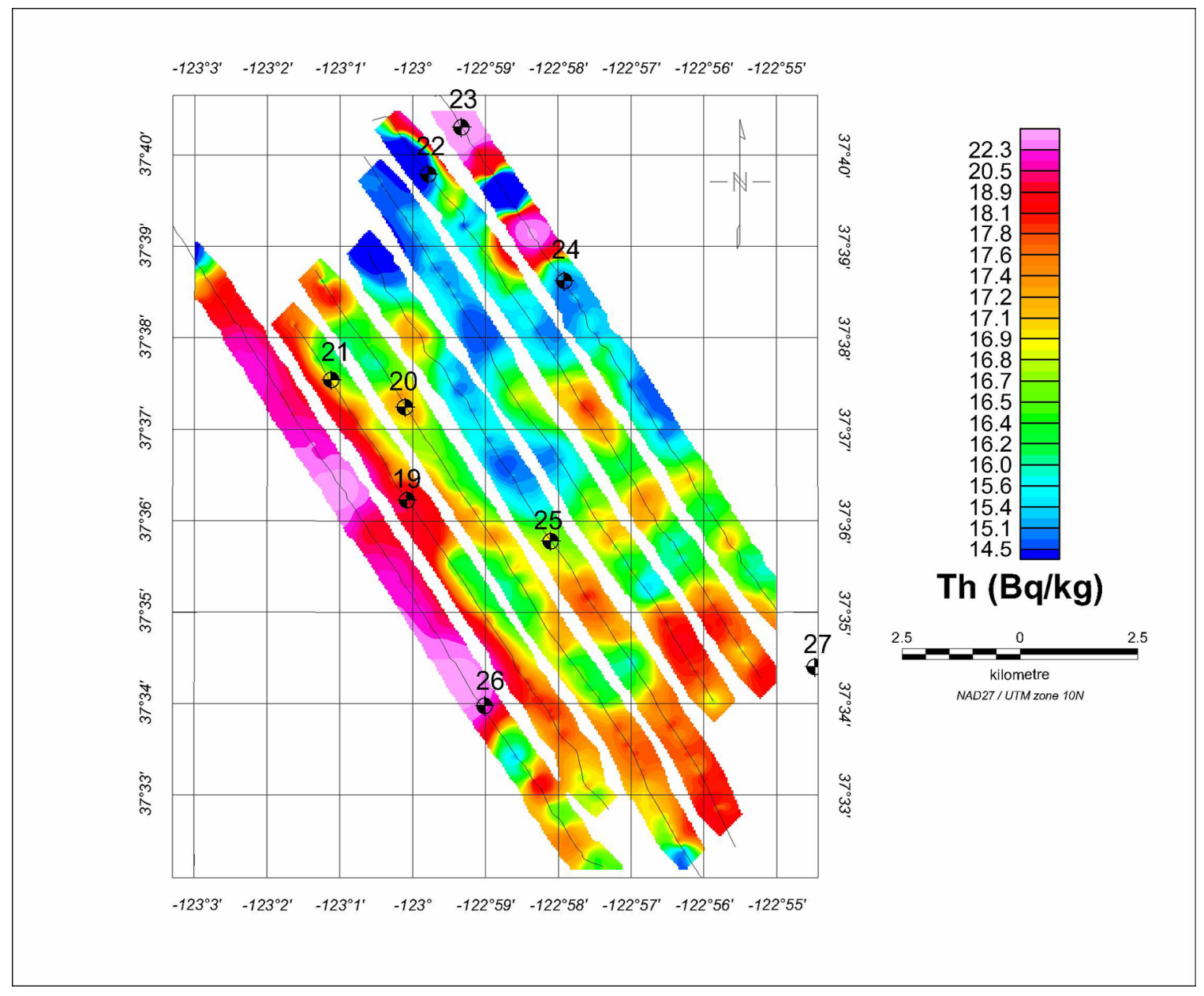

Figure 19. Th data for the $90-\mathrm{m}$ site. Typical uncertainties $(1 \sigma)$ better than 5 percent.

radionuclides from the barrels of waste, but this only seems to have raised radionuclide levels slightly in the sediments in the vicinity of the barrels. There is no evidence for significant regional-scale contamination as a result of the dumping in the areas surveyed.

Levels of natural radionuclides are well within the normal range for sea floor sediments, although there could have been some input of $\mathrm{U}$ and $\mathrm{Th}$ from the barrels.

It should be borne in mind, however, that, at this stage, no data have been obtained for large areas of the dump site. In particular the $1800 \mathrm{~m}$ site, where the majority of the barrels are believed to have been dumped, remains virtually unstudied, in terms of both the radionuclide content of the sediments and the actual locations of the barrels. To date barrel locations have only been mapped in $15 \%$ of the dump area, and radionuclide concentrations examined in only about $10 \%$ of the site. On the other hand, the areas studied are those in the shallower waters of the $90-\mathrm{m}$ and $900-\mathrm{m}$ sites most accessible to man.

This study, along with the earlier USGS work, shows the value of interagency co-operation and the merit of being able to target measurements and sampling on the basis of knowledge of barrel locations. This integrated approach could be extended to the deeper parts of this site and applied in other areas, such as Boston Harbour, the Kara Sea, and Beauforts Dyke in the Irish Sea. The data obtained by this type of study enable informed decisions to be made regarding the environmental quality and management of the ocean. 


\section{Acknowledgments}

We are grateful to M.H. Strutt, D.K. Talbot, M P Rainey and J.R. Davis, of the BGS for logistical support, sample analysis, data processing and presentation. The assistance of the USGS Marine Facility staff (S. Wallace, D. Hogg, L. Kooker, F. Payne and others) is acknowledged with thanks. Navigational support was provided by M. Hamer and G. Dunhill of the USGS and radiological safety support by C. Petullo, L. Karr and S. Faller of EPA. The work would not have been possible without the help of the officers and crew of the NOAA ship McArthur and the co-operation of the Gulf of the Farallones National Marine Sanctuary.

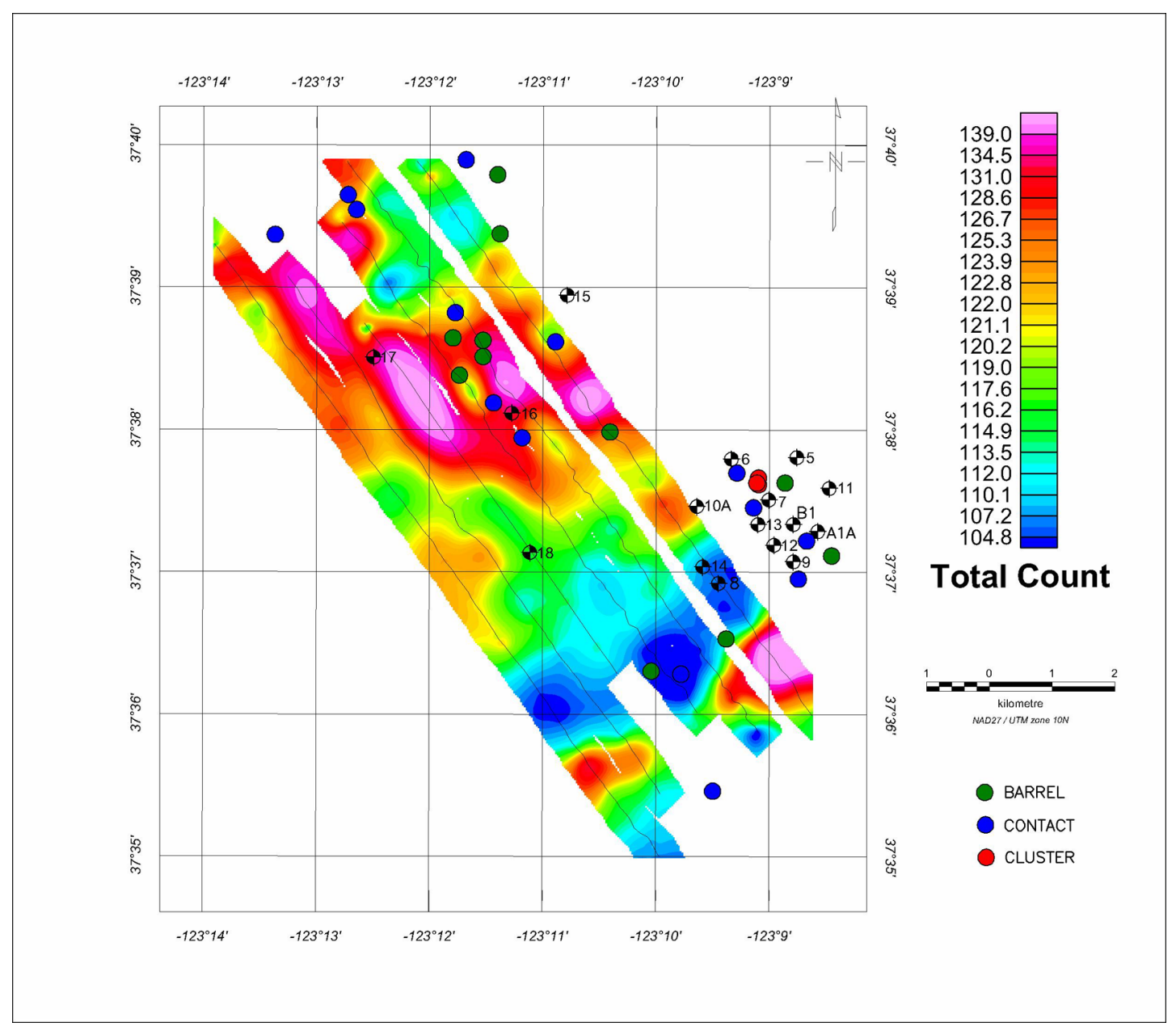

Figure 20. Total count data for the $900-\mathrm{m}$ site $\left(\right.$ counts. $\left.\mathrm{s}^{-1}\right)$. Typical uncertainties $(1 \sigma)$ better than 1 percent. 


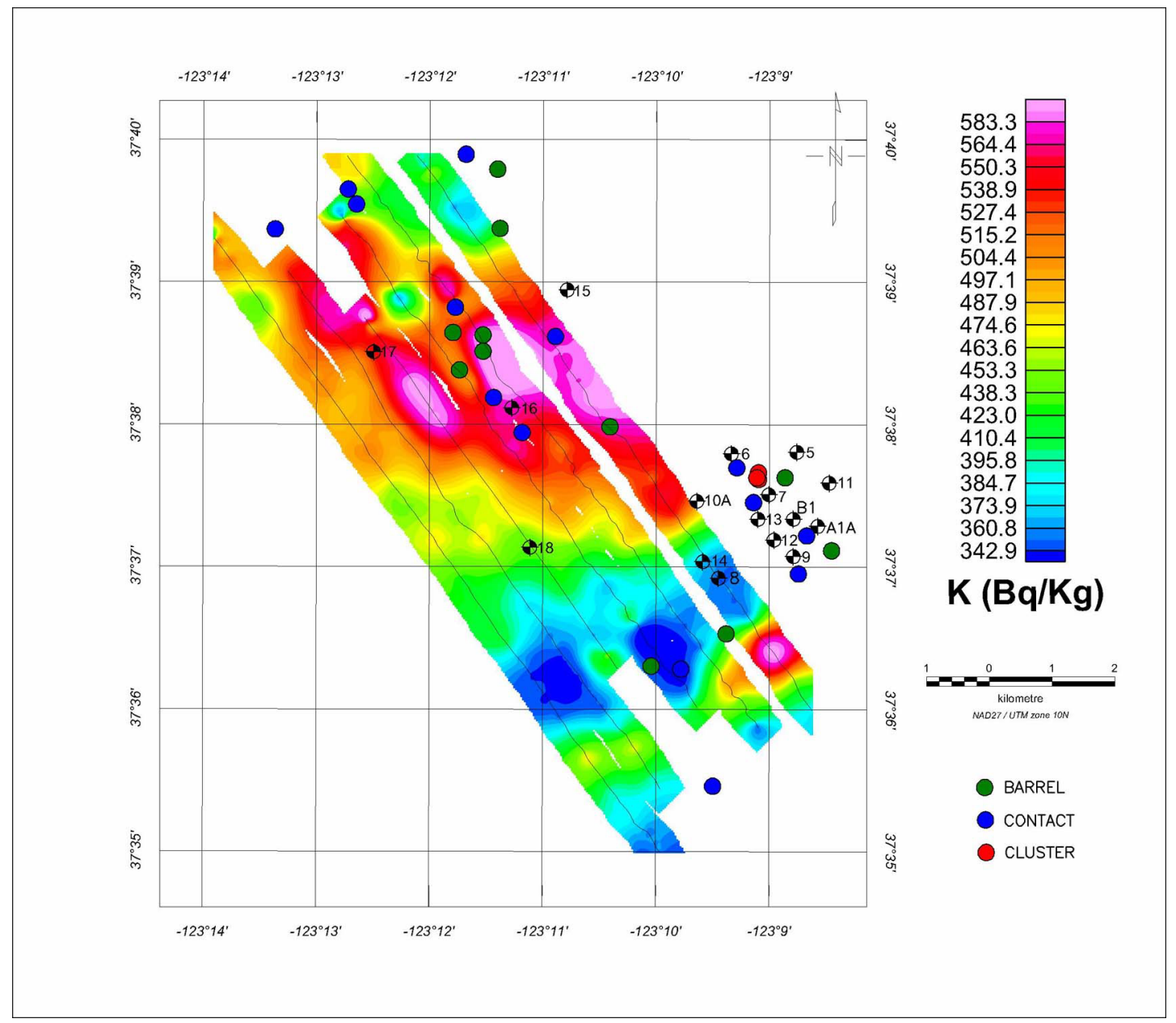

Figure 21. K data for the 900-m site. Typical uncertainties $(1 \sigma)$ better than 2 percent. 


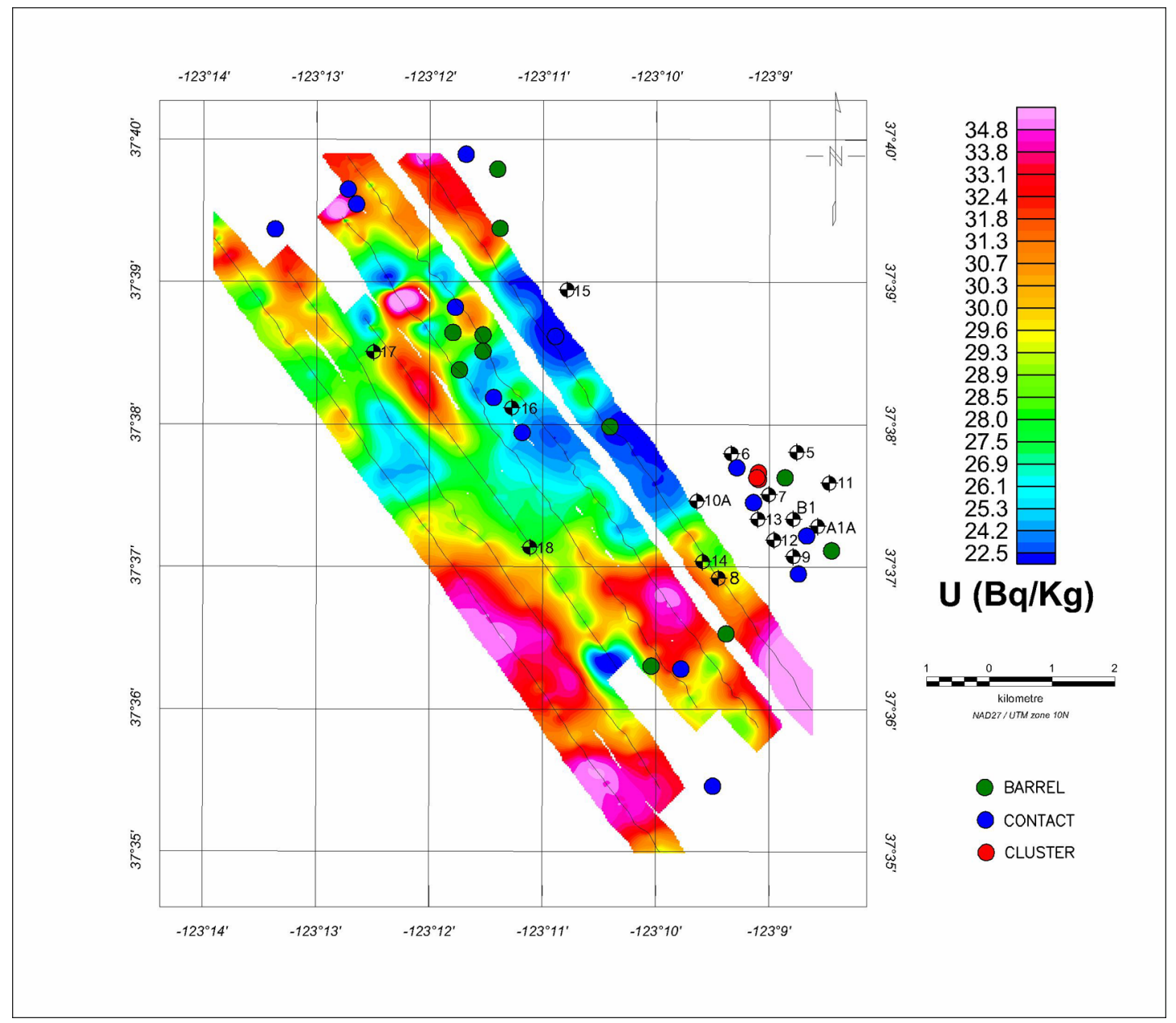

Figure 22. U data for the $900-\mathrm{m}$ site. Typical uncertainties $(1 \sigma)$ better than 25 percent. 


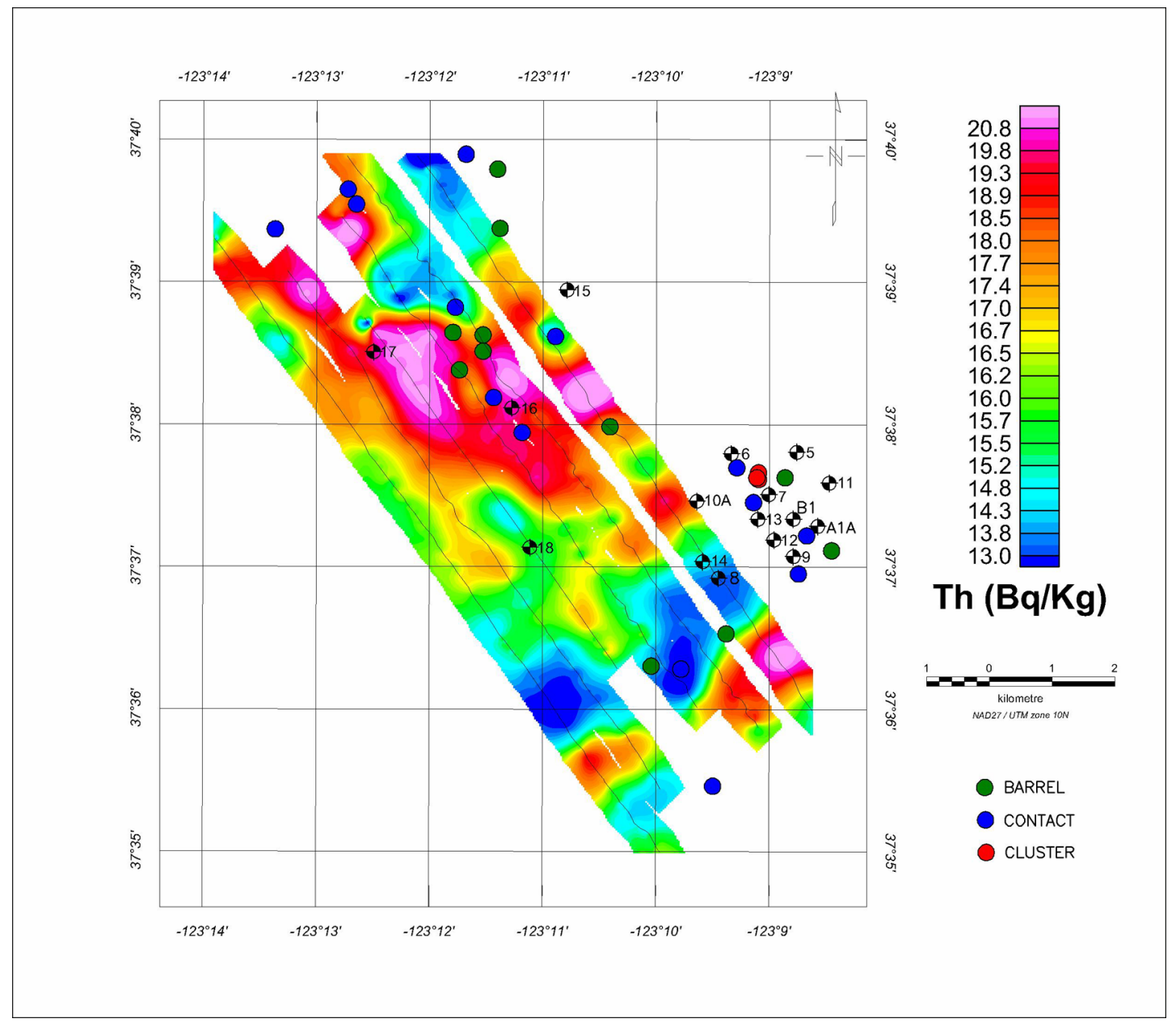

Figure 23. The data for the $900-\mathrm{m}$ site. Typical uncertainties $(1 \sigma)$ better than 5 percent. 


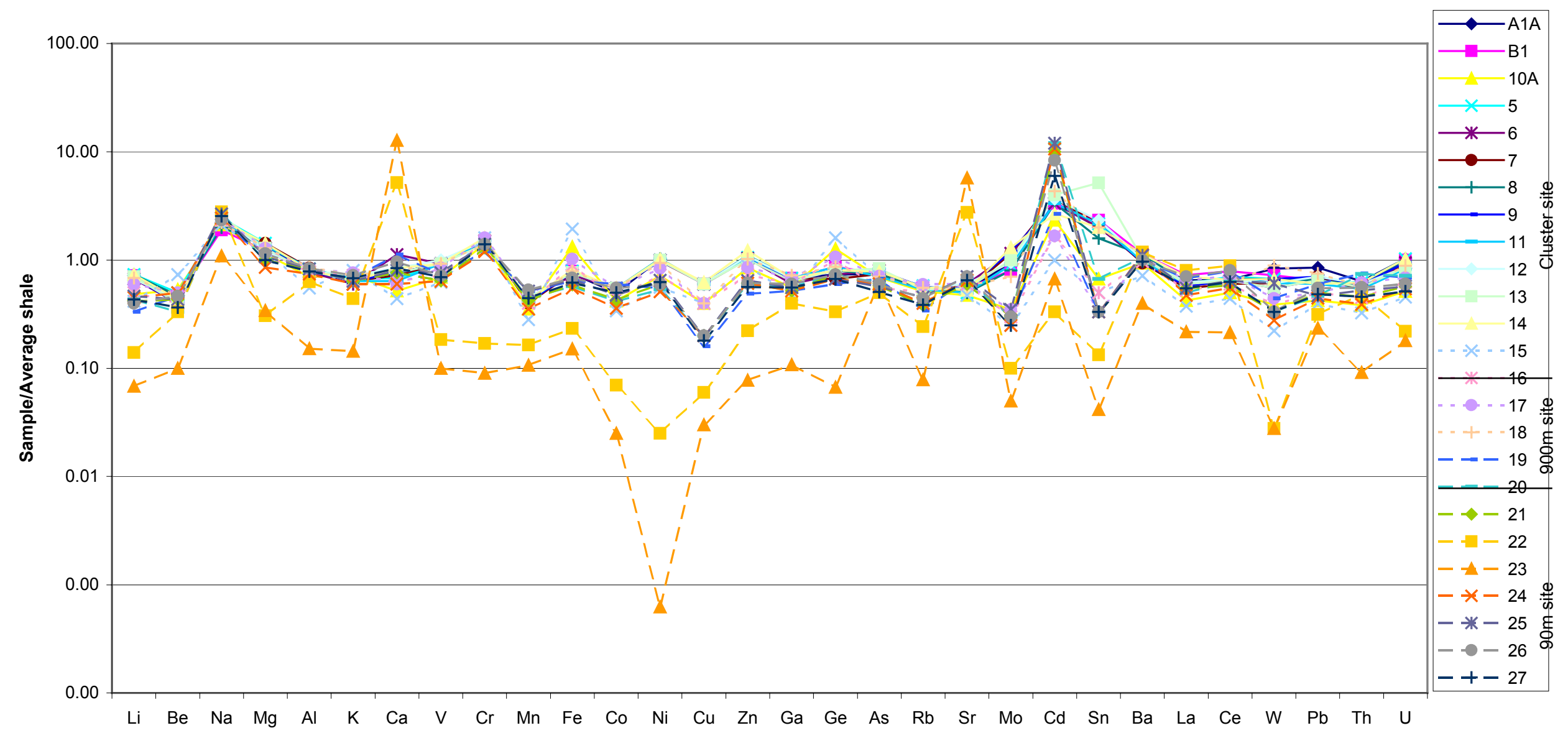

Figure 24A. Multielement geochemical plots for sediment samples. All samples. 


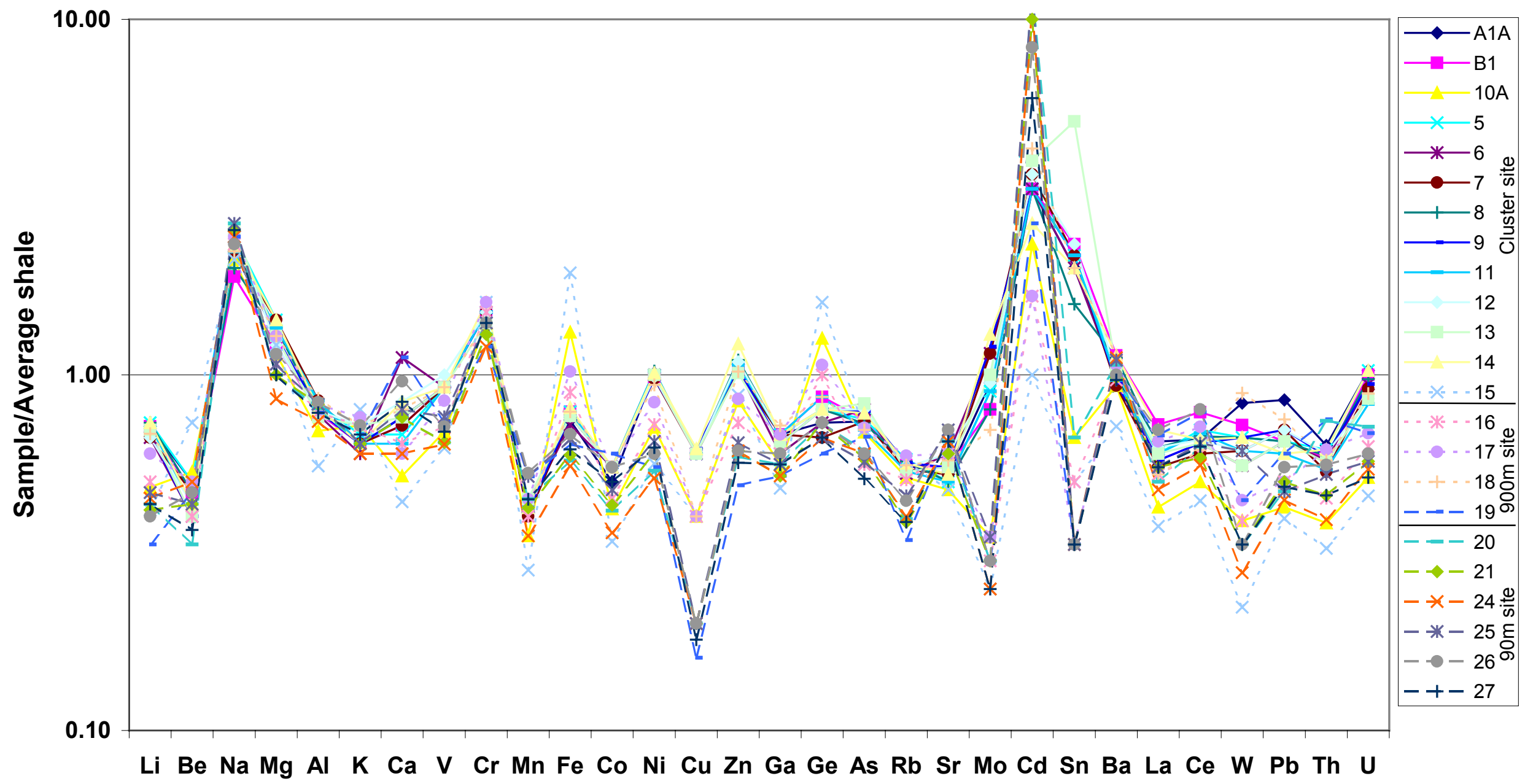

Figure 24B. Multielement geochemical plots for sediment samples. Two shelly samples $(22,23)$ omitted. 


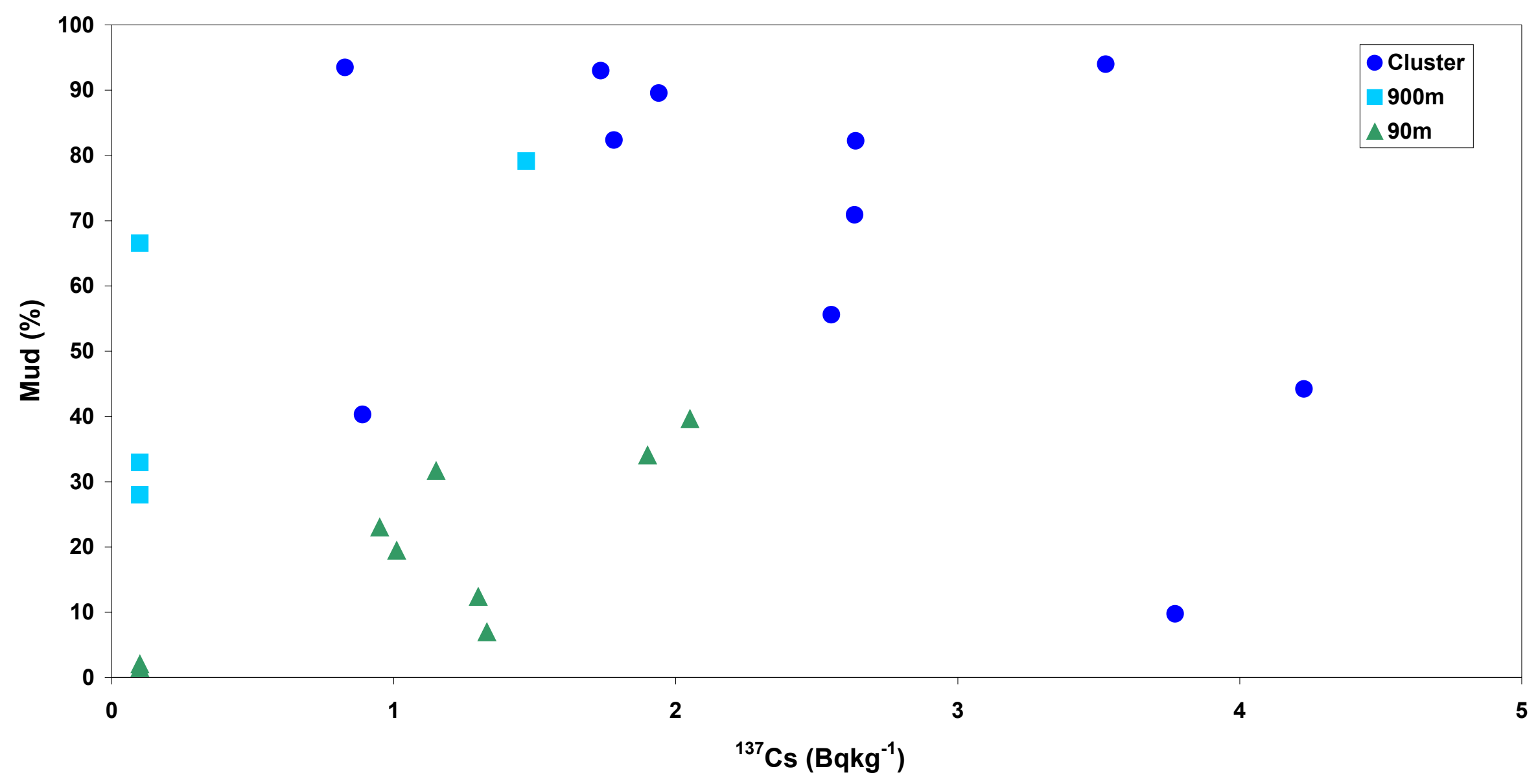

Figure 25. Relationship of ${ }^{137} \mathrm{Cs}$ to mud content of sediments samples. 


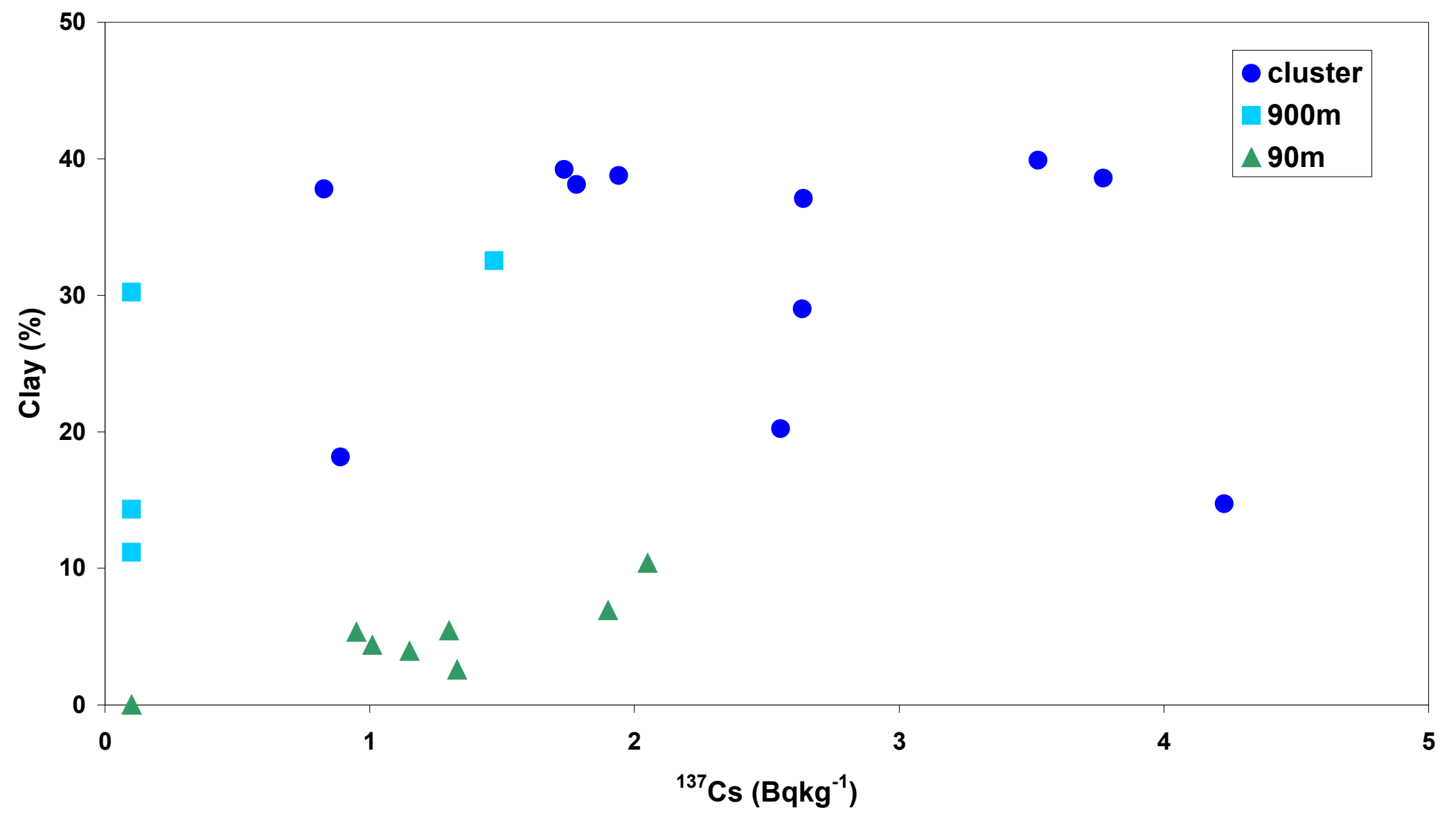

Figure 26. Relationship of ${ }^{137} \mathrm{Cs}$ to clay content of sediments samples. 


\section{References}

Booth, J. S., Winters, W. J., Poppe, L. J., Neiheisel, J. \& Dyer, R. S. (1989) Geotechnical, Geological, and Selected Radionuclide Retention Characteristics of the Radioactive-Waste Disposal Site Near the Farallon Islands. Marine Geotechnology, 8, 111-132.

Briggs, P. H. \& Meier, A. L. (1999) The determination of forty-two elements in geological materials by inductively coupled plasma-mass spectrometry. U.S. Geological Survey Open File Report, 99-166.

de Meijer, R. J., Stapel, C., Jones, D. G., Roberts, P. D., Rozendaal, A. \& Macdonald, W. G. (1997) Improved and new uses of natural radioactivity in mineral exploration and processing. Exploration and Mining Geology, 6, 105-117.

Dyer, R. S. (1976) Environmental surveys of two deep-sea radioactive waste disposal sites using submersibles. In: Proceedings of the International Symposium on the Management of Radioactive Wastes from the Nuclear Fuel Cycle, pp. 317-338. IAEA, Vienna.

FSA/SEPA. (2000) Radioactivity in food and the environment, 1999. Food Standards Agency/Scottish Environmental Protection Agency Report, RIFE - 5.

Griggs, G. B. \& Hein, J. R. (1980) Sources, dispersal, and clay mineral composition of fine-grained sediment off central and northern California. Journal of Geology, 88, 541-566.

Hendriks, P. H. G. M., Limburg, J. \& de Meijer, R. J. (2001) Full spectrum analysis of natural gamma ray spectra. Journal of Environmental Radioactivity, 53, 365-380.

Jones, D. G. (1994) Towed Seabed Gamma-Ray Spectrometer. Sea Technology, 35, 89-93.

Jones, D. G., Miller, J. M. \& Roberts, P. D. (1988a) A seabed radiometric survey of Haig Fras, S. Celtic Sea, U.K. Proceedings of the Geologists' Association, 99, 193-203.

Jones, D. G., Roberts, P. D. \& Miller, J. M. (1988b) The Distribution of Gamma-Emitting Radionuclides in Surface Subtidal Sediments Near the Sellafield Plant. Estuarine Coastal and Shelf Science, 27, 143-161.

Jones, D. G., Roberts, P. D., Strutt, M. H., Higgo, J. J. \& Davis, J. R. (1999) Distribution of Cs-137 and inventories of Pu238, Pu-239/240, Am-241 and Cs-137 in Irish Sea intertidal sediments. Journal of Environmental Radioactivity, 44, 159-189.

Joseph, A. B., Gustafson, P. F., Russell, I. R., Schuert, E. A., Volchok, H. A. \& Tamplin, A. (1971) Sources of radioactivity and their characteristics. In: Radioactivity in the marine environment, pp. 6-41. National Academy of Sciences, Washington, D.C.

Karl, H. A., Schwab, W. C., Wright, A. S. C., Drake, D. E., Chin, J. L., Danforth, W. W. \& Ueber, E. (1994) Acoustic mapping as an environmental management tool: I. Detection of barrels of low-level radioactive waste, Gulf of the Farallones National Marine Sanctuary, California. Ocean and Coastal Management, 22, 201-227.

Kershaw, P. J., Denoon, D. C. \& Woodhead, D. S. (1999) Observations on the redistribution of plutonium and americium in the Irish Sea sediments, 1978 to 1996: concentrations and inventories. Journal of Environmental Radioactivity, $44,191-221$.

Miller, J. M., Roberts, P. D., Symons, G. D., Merrill, N. H. \& Wormald, M. R. (1977) A towed gamma-ray spectrometer for continental shelf surveys. In: International Symposium on Nuclear Techniques in Exploration, Extraction and Processing of Mineral Resources, pp. 465-498. IAEA, Vienna.

Miller, J. M., Thomas, B. W., Roberts, P. D. \& Creamer, S. C. (1982) Measurement of Marine Radionuclide Distribution Using a Towed Sea-Bed Spectrometer. Marine Pollution Bulletin, 13, 315-319.

NOAA. (1990) Farallon Islands Radioactive Waste Dumps, NOAA Preliminary natural resource survey.

Noshkin, V. E., Wong, K. M., Jokela, T. A., Eagle, R. J. \& Brunk, J. L. (1978) Radionuclides in the marine environment near the Farallon Islands. Lawrence Livermore Laboratory, University of California Report, UCRL-52381.

PneumoDynamics Corporation. (1961) Survey of radioactive waste disposal sites. PneumoDynamics Corporation Advanced Systems Development DivisionTechnical Report, ASD 4634-F. 
Ringis, J., Jones, D. G., Roberts, P. D. \& Caringal, R. R. (1993) Offshore geophysical investigations, including use of a sea bed gamma spectrometer, for heavy minerals in Imuruan Bay, N W Palawan, S W Philippines. British Geological Survey Technical Report, WC/92/65, 54pp.

Schell, W. R. \& Sugai, S. (1980) Radionuclides at the U.S. radioactive waste disposal site near the Farallon Islands. Health Physics, 39, 475-496.

Suchanek, T. H., LagunasSolar, M. C., Raabe, 0. G., Helm, R. C., Gielow, F., Peek, N. \& Carvacho, 0. (1996) Radionuclides in fishes and mussels from the Farallon islands nuclear waste dump site, California. Health Physics, 71, 167-178.

Turekian, K. K. \& Wedepohl, K. H. (1961) Distribution of the elements in some major units of the Earth's crust. Bulletin of the Geological Society of America, 72, 175-192.

Waldichuk, M. (1960) Containment of radioactive waste for sea disposal and fisheries off the Canadian Pacific coast. In: Proceedings of IAEA Symposium on Disposal of Radioactive Wastes, Vol. 2. IAEA, Vienna.

Wedepohl, K. H. (1978) Handbook of Geochemistry. Springer-Verlag, Berlin. 